\title{
POLY(LACTIC ACID) FOR DELIVERY OF BIOACTIVE MACROMOLECULES
}

Roshan James $^{1,2, \dagger}$, Ohan S. Manoukian ${ }^{1,3, \dagger}$, Sangamesh G. Kumbar ${ }^{1,2,3,4^{*}}$ 


\begin{abstract}
Therapeutic biomolecules often require frequent administration and supramolecular dosing to achieve therapeutic efficiencies and direct infusion into treatment or defect sites results in inadequate physiological response and at times severe side effects or mis-targeting. Delivery systems serve several purposes such as increased circulatory time, increased biomolecule halflife, and incorporation of new innovations can enable highly specific cell targeting and improved cell and nucleus permeability. Poly(lactic acid) (PLA) has become a "material of choice" due to wide availability, reproducible synthetic route, customization, versatility, biodegradability and biocompatibility. Furthermore, PLA is amenable to a variety of fabrication methodologies and chemistries allowing an expansive library correlating physio-chemical properties, characteristics, and applications. This article discusses challenges to biomolecule delivery, and classical approaches of PLA based biomolecule delivery and targeting strategies under development and in trials.
\end{abstract}

Keywords: Biomolecule, Peptide, DNA, RNA, Protein, Nanoparticle, Targeting, Internalization, Blood-Brain Barrier,

Chemical compounds studied in this article

Polylactic acid (PubChem CID: 612); Polyethylene glycol (PubChem CID: 174); Clozapine (PubChem CID: 2818); Penetratin (PubChem CID: 16134279); Thyrotropin-releasing hormone (PubChem CID: 32281); Paclitaxel (PubChem CID: 36314); Doxorubicin (PubChem CID: 31703); NGR peptide ((PubChem CID: 18219107); polydimethylaminoethyl methacrylate (PubChem CID: 107676) 


\section{Graphical Abstract:}

Biomolecules

DNA/RNA

Specificity Peptide

Protein

Cell Penetrating Protein

Peptide Conjugate

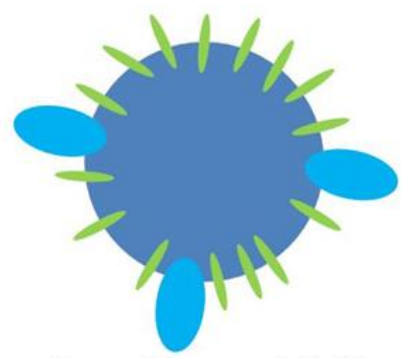

Targeting and Cell
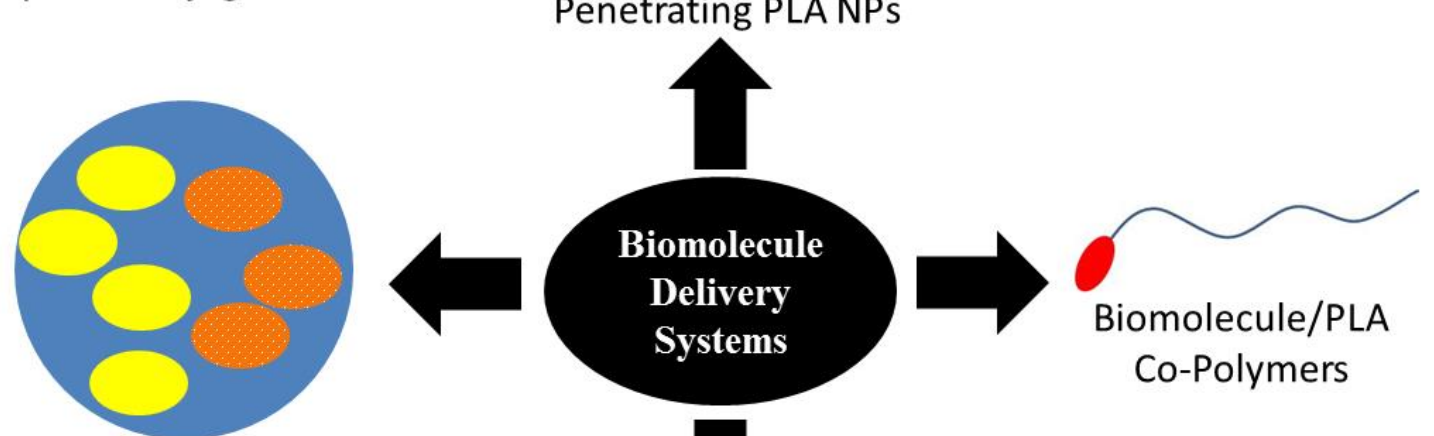

Biomolecule encapsulated PLA NPS

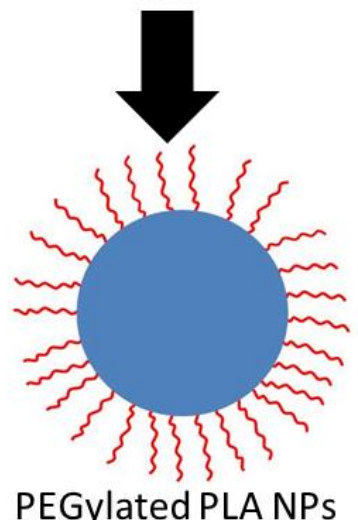




\section{Introduction}

Controlled drug delivery strategies continue to be the most convenient and compliant means of patient treatment. The rapid development of biotechnology techniques has promoted large-scale production and synthesis of biomolecules, and they have demonstrated significant potential against many diseases, and have application in cutting edge areas including diagnostics and rapid analysis [1-5]. They are an important class of therapeutic drugs today over other chemical drugs due to their advantages of low side effects and specific targeting abilities [6]. However, the biological nature of these drugs and molecules pose a significant challenge in navigating the native defense mechanisms in our body, as they are characterized by high susceptibility to hydrolysis, enzymatic degradation and short in vivo half-life requiring frequent dosing. Researchers have aggressively tackled many of the issues involved in controlled release and delivery of biomolecules by innovating techniques to protect the biomolecule from degradation mechanisms, metered release of therapeutic concentrations to ensure high serum bioavailability, and by incorporating targeting approaches to minimize systemic toxicity.

Biodegradable polymers are significantly advantageous to non-degradable polymers which require a secondary procedure for removal. Poly(lactic acid) is among the most widely used biodegradable polymer and combined with properties of low immunogenicity and toxicity, it is highly biocompatible and can be reproducibly fabricated as compared to polymers derived from natural sources. Physicochemical and mechanical properties of PLA can be tuned to the necessary application requirements through appropriate selection of molecular weight, copolymerization with other polymers, and functionalization to impart desired features [7-14]. Furthermore, researchers have engineered diverse fabrication methodologies to entrap therapeutics, ensure controlled release and achieve targeting capabilities for diverse drugs and biomolecules leading to several pharmaceutical products, including vaccines, anti-inflammatory and antagonistic treatments. This article focuses on the delivery of biomolecule agents, identifying their challenges and examining the innovative approaches to delivery, target and controlled release from PLA polymers. Numerous fabrication methods for biomacromolecule delivery systems using PLA based polymers have been reported including emulsification, spray 
drying, electrospraying, nanoprecipitation, self-assembly, pegylatation and supercritical methods and described in detail elsewhere $[8,9,15,16]$.

\section{Challenges in Developing Biomacromolecule Delivery Systems}

The field of biomacromolecular therapy has made tremendous progress over the last decade due to our improved understanding of drug properties and cellular uptake mechanisms, along with the development of controlled release systems and strategies to improve stability of the therapeutic agents. Despite their conceptual simplicity, several challenges and technical barriers to the development of a successful delivery mechanism for biomacromolecular therapeutics have emerged. Macromolecular therapeutic agents suffer from stability and bio-distribution issues in vivo, with their physical and chemical makeup making them particularly vulnerable to enzymatic and chemical influences including degradation, deactivation, and excretion. A key issue for the future growth and evolution of biomacromolecular therapies and therapeutics is the efficient and effective delivery of small and large biomolecules (e.g. peptides, proteins, genes, siRNA) to the target areas. Major challenges to the delivery of macromolecules include rapid renal clearance from the body, intracellular nuclease mediated degradation, and cell membrane permeability, all of which reduce the efficiency and effectiveness of macromolecular drug delivery.

\subsection{Rapid Renal Clearance}

Low molecular weight macromolecules, specifically oligonucleotides and DNA/RNA aptamers, provide significant advantages such as an economical chemical synthetic route and greater targeting abilities. However, it also facilitates their rapid elimination from the body by renal clearance. Along with toxins, unmetabolized nutrients, minerals, and ions, such macromolecules are passed through the kidney and taken up by the glomerular or peritubular capillaries and eventually eliminated from the body by renal clearance. Important factors governing the rate of filtration and renal clearance are the molecular weight and electrical charge of the macromolecules. Small molecules often experience rapid renal clearance, with plasma half-life anywhere from a couple minutes to approximately 30 minutes [17]. Furthermore, negatively charged macromolecules have been shown to be filtered to a lesser extent than neutral molecules, 
because the renal tubule membrane surface is composed of polyanions which repel negatively charged macromolecules [18].

As such, the bioavailability of macromolecule agents can be extended by increasing the molecular weight. One common method is the conjugation of the molecule to polyethylene glycol (PEG) in a process known as PEGylation. The attachment of high molecular weight PEG is widely used with DNA/RNA aptamers, oligonucleotides, proteins, and peptides to slow down renal clearance and extend half-life and bioavailability. Healy et al., conclusively demonstrated the circulation half-life of a 40kDa PEGylated aptamer was significantly longer than a $20 \mathrm{kDa}$ PEGylated aptamer of the same base composition, with the 40kDa PEG-conjugate having a halflife of 12 hours, as compared to the 20KDa PEG-conjugate having a half-life of nearly 7 hours, and the unconjugated aptamer having a half-life of 5 hours. [19]. Alternative methods to reduce renal clearance of drugs and macromolecules include the encapsulation of drug molecules in nanoparticles and altering polymer structures. Kadam et al. demonstrated the effects of nanoparticle encapsulation on the clearance of Clozapine, a lipophilic molecule which undergoes extensive first-pass metabolism resulting in poor bioavailability and a half-life of about 4.8 hours [20]. Clozapine nanoparticles showed 3-fold lower clearance than plain drug suspension with a significantly longer half-life of 8.7 hours. Surface properties and structures have also been shown to influence renal clearance [20-22]. Following drug administration through the oral route, positively charged clozapine nanoparticles exhibited 3.0 to 5.3-fold longer circulation times (half-life) than negative and neutral nanoparticles. Furthermore, Levchenko et al. showed that negatively charged liposomes have atleast $80 \%$ higher plasma clearance than those of neutral charge with neutral [22].

\subsection{Intracellular Nuclease Mediated Degradation}

In addition to rapid renal clearance, nuclease mediated degradation of biomacromolecules is a significant challenge that must be overcome to delivery biomacromolecules to the intended intracellular targets. Unmodified or unprotected DNA/RNA aptamers, oligonucleotides, proteins,

and peptides are almost immediately degraded and metabolized when they enter the cell by nucleases and/or enzymes. Incorporation of modifications of the sugar moieties and inter- 
nucleotide phosphodiester linkages can help to extend half-life of macromolecules. The incorporation of fluoro- and O-methyl groups specifically at the 2' position of DNA/RNA sugar moieties enhances physicochemical stability of the biomacromolecules and in combination with polymer approaches can protect the therapeutic agents extending their half-life in blood. Both nuclease degradation and renal clearance affect the bio-distribution and bioavailability of macromolecular agents, presenting unique challenges for efficient and effective in vivo delivery [23].

\subsection{Cell Membrane Permeability}

Cellular membranes exhibit exquisite regulation of molecular and ion transport both in and out of the cell. They are, in general, permeable to smaller molecules, such as sugars and small ions, but are rather impermeable to large, polar molecules, such as proteins and nucleic acids [24]. Thus, macromolecules are inherently faced with limitations as most will fail to permeate cell membranes, presenting a formidable problem for the effect delivery to targets located within the cell. Inspired by viruses and bacterial macromolecules which possess capabilities to cross the cell membrane [24]; novel viral macromolecules have been developed to deliver proteins and nucleic acid payloads into the cytoplasm and the cell nucleus. Decoding the sequences of viral proteins have led to the development of cell penetrating peptides (CPPs) which can be conjugated to therapeutic peptides, oligonucleotides, and proteins allowing them to penetrate the cellular membrane, which otherwise is considered non-permeable. CPPs are largely divided into two classes: the first consist of amphipathic helical peptides where lysine contributes positive charge, e.g. include transportan and model amphipathic peptide (MAP), and the second class includes arginine-rich peptides such as TAT and penetratin [25-27]. CPPs have been developed for the transport of covalently and non-covalently linked cargo across cellular membranes including nanoparticles, peptides, proteins, antisense oligonucleotides, siRNA, DNA, and liposomes [26, 28-32]. Uptake of CPPs across the cellular membrane to the cell interior occurs either by direct endocytosis or by direct penetration by mechanisms such as interactions with cell membrane sulfates, cell surface receptors, pinocytosis, and clathrin and caveolae-mediated endocytosis [33]. Xia et. al. reported the fabrication of penetratin conjugated PEG-PLA nanoparticles via a maleimide thiol coupling reaction [34]. In vivo pharmacokinetic and biodistribution studies 
showed that penetratin-PEG-PLA nanoparticles exhibited a significantly enhanced brain uptake and reduced accumulation in the non-target tissues organs indicating a promising brain-targeting drug delivery system. In the presence of serum proteins which are negatively charged, the positively charged nanoparticles tend to form aggregates thereby exhibiting rapid clearance and accumulation in tissues such as the lung and liver, which was significantly minimized and to a large extent avoided by penetratin-PEG-PLA nanoparticles which have a seta potential of -4.42 $\mathrm{mV}$.

\subsection{Binding-Site Barrier}

High affinity of biomacromolecules to the target receptor will enable specific and targeted delivery reducing dosage requirements to achieve therapeutic efficacy. However, several theories suggest that high-affinity molecules despite successful binding may be limited to the periphery of the target tissue [35-37]. The high-affinity macromolecules will bind to target receptors immediately adjacent or within close proximity to the blood vessels, which may limit their binding to other more distant receptor sites, leading to poor drug penetration of the target tissue or organ. The resulting binding-site barrier leads to a heterogeneous therapeutic distribution throughout the target tissue and reduced effectiveness due to incomplete penetration of the biomacromolecular therapeutic agent [36].

\subsection{Toxicity and Off-target Interactions}

Toxicity and therapeutic efficiency are critical characteristics of every successful drug therapy, and studies have reported immune response stimulation on treatment with oligonucleotide and RNA aptamer agents, observed through hyperplasmia and mononuclear cell infiltration [23]. High concentrations of oligonucleotide agents or antisense oligonucleotides as well as nonspecific or off-target proteins can produce what is known as the polyanion effect. In primates, oligonucleotide aggregation has been shown to cause activation of an alternative pathway labeled 'C', leading to hypersensitivity, hypotension, tachycardia, and inhibition of critical blood coagulation networks [23]. Other off-target interactions can lead to unwanted, or perhaps even 
uncontrollable, reactions, much of which is not well understood and remains a primary focus of clinical safety trials [24].

\section{Bioactive Macromolecules - Roles and Therapeutic Strategies}

Advances in molecular and cell biology have led to the discovery and subsequent development of various classes of biomacromolecules including antisense oligonucleotides, nucleic acid and peptide aptamers, genes, enzymes, proteins and interfering RNAs which have been investigated for their therapeutic efficacy and physicochemical properties in vivo using controlled release systems, and for applications such as biosensors, diagnostic assays, microarrays, analytical probes, and other procedures. These complex molecules are alternatives to traditional chemicals, small-molecular weight drugs as safety and efficacy is enhanced by their superb specificity, selectivity, and targeting capabilities [38-40].

\subsection{Nucleic Acid and Peptide Aptamers, and Therapeutics}

Nucleic acid and peptide aptamers are molecules with three-dimensional (3D) structure, engineered and isolated from large oligomer libraries. Their molecular recognition properties, engineering capabilities, and relatively simple synthetic route and storage make them advantageous over more commonly used, traditional therapeutics such as antibodies. Nucleic acid aptamers, either DNA or RNA based, are typically selected using an in vitro evolution methodology based on combinatorial chemistry technique referred to as SELEX (Systematic Evolution of Ligands by Exponential enrichment) [24]. A large working library of oligonucleotides is created and candidate molecules are screened for specific affinities and binding to various targets. The screening process involves exposure of the library to the target of interest, which may be a protein, nucleic acid, or small molecule. Molecules that successfully bind to the target form complexes, which are then recovered via affinity purification and amplified using polymerase chain reaction (PCR). Numerous iterative rounds of selection leads to a purified pool of aptamers having very high binding affinities and selectivity to the target [41]. This process is represented schematically in Figure 1. 
Peptides are short chain amino acids (typically less than 100 residues) linked together by peptide bonds, and are similarly screened for high-affinity candidates toward a particular target of interest. Three primary approaches exist for the identification and selection of high-affinity peptide candidates (Figure 2). In the first approach, a library of peptides is exposed to an immobilized target of interest (peptide screen). In the second approach, the reciprocal is true, where a peptide species is immobilized and a library of targets is exposed to the peptide of interest (target screen). Finally, bacteriophages expressing various peptides on their surface are exposed to an immobilized target of interest and analyzed for their affinity (phage display) [42]. In phage sequence, the peptide DNA is contained within the respective bacteriophage, allowing for straightforward identification of the peptide [24]. There has been significant development in the area of therapeutic peptides and is evidenced by the nearly 600 peptides that are currently under various stages of pre-clinical trials [42], with promising results in treating or controlling diseases such as type II diabetes, vaccines and anti-microbial medications [43]. Upon binding, aptamers can specifically inhibit the target's functions and therapeutically, they are most commonly used with extracellular targets such as thrombin (blood clotting cascade) [41], circulating tumor cells [44], and monocyte proteins for type II diabetes [45].

Researchers have turned to polymeric drug delivery approaches such as nanoparticles (NPs) for improved delivery of peptide therapeutics reducing the risks of degradation, renal excretion, and limited cellular permeability. Veronesi et al., reported the use of poly (lactic acid) (PLA) intranasal NPs delivery system containing thyrotropin-releasing hormone (TRH) to the central nervous system (CNS). TRH is a peptide hormone produced by the hypothalamus and reported to have anticonvulsant effects in seizure models and epileptic patients. Traditional mechanisms of TRH delivery are limited by rapid metabolism and poor permeability of the blood-brain barrier, resulting in ineffective therapeutic concentrations. Intra-nasal administration of TRH NPs presents an alternative, and shorter, route of administration to the brain. The use of PLA NPs combated rapid metabolism of the neuropeptide and facilitated its transport across the blood-brain barrier. Results indicated neuroprotective properties of TRH NPs against Glutamate toxicity in vitro, while significantly raising the stimulations required to produce stage $\mathrm{V}$ seizures and suppressing seizure characteristics in in vivo rat models [46]. 
Tumor-specific peptides have been developed and are a rapidly growing area, particularly for the treatment of glioma which accounts for roughly $43 \%$ of all brain tumors and is highly proliferative and invasive, requiring a combination of aggressive chemotherapy, radiation, and surgical removal $[47,48]$. Drug delivery to glioma is severely limited by non-specific therapeutic agents and the inability to permeate across the blood-brain barrier, as well as the blood-brain tumor barrier. Utilization of active-targeting, tumor-specific antibodies and peptides overcome some of the aforementioned shortcomings. However, these approaches lack effective cell membrane permeation and cellular internalization. Thus, a homogenous distribution into the glioma stands as a major obstacle to anti-glioma peptide delivery [49]. Taking advantage of specific markers highly expressed on the surface of glioma cells and glioma angiogenic blood vessels, Hu et al., utilized PEG-PLA NPs as a drug delivery system (DDS) to co-administer two peptides to mediate efficient tumor targeting and internalization. F3 peptide which specifically binds to the highly-expressed nucleolin marker on glioma cells was used in conjunction with Lyp-1 peptide, a tumor homing and penetrating peptide, to decorate the PEG-PLA NPs. In vitro studies showed the F3-NP complex distributed more extensively in tumor spheroids than the control NPs alone. The penetration ability was quantified by depth, revealing F3-NP penetrated 139.26 $\mu \mathrm{m}$, while the control NPs penetrated $81.02 \mu \mathrm{m}$ [49]. In vivo biodistribution and antiglioma efficiency was studied, and it showed that F3-NP co-administrations resulted in higher fluorescent intensity at the tumor sites than control NPs. F3-NP also showed superior accumulation at glioma sites compared to controls, confirming the ability of co-administration to overcome internalization and permeability limitations (Figure 3). When loaded with paclitaxel (PTX), a model chemotherapeutic drug, the longest survival in mice with C6 glioma was achieved by F3-NP co-administrations, with a median survival of 42 days which was significantly higher than other treatments and controls [48]. As such, these results suggest that the F3-NP co-administration DDS enhanced permeation across the blood-brain barrier and the blood-brain tumor barrier, as well as glioma-specific cellular internalization and penetration.

PLA DDSs have also been used as vehicles for peptide therapeutics in ocular illnesses [50]. Hyuncheol et al., investigated the delivery of angiogenesis inhibitor peptide C16Y using PLA/PLA-PEO NPs to the sub-retinal space to combat choroidal neovascularization, a major cause of severe vision loss associated with age-related macular degeneration. Long diffusion 
distances, rapid clearance by aqueous humor and sclera, and ineffective retinal penetration pose challenges for effective topical and trans-scleral drug delivery [50]. The C16Y peptide has been shown to inhibit neovascularization and reduce chorioallantoic angiogenesis and tumor growth. The C16Y peptide has a short physiological half-life necessitating a different means for storage and sustained release. Utilizing PLA/PLA-PEO NPs, researchers were able to protect the C16Y peptide from rapid diffusion and clearance. In vitro release studies showed sustained release characteristics of the encapsulated C16Y peptide over 6 weeks, when 19mg of NPs containing $1.07 \mathrm{mg}$ of $\mathrm{C} 16 \mathrm{Y}$ were placed in pure water [50]. In vivo models revealed interesting results when C16Y-NPs were compared to C16Y peptide solution. Although the C16Y peptide solution delivered 25 times more peptide when injected than C16Y-NPs, choroidal neovascularization was inhibited more with the C16Y-NPs than the peptide solution [50]. Although both groups performed better than placebo PLA/PLA-PEO NP controls, the short half-life of intravitreal C16Y combined with rapid clearance disallowed the peptide solution from inhibiting experimental choroidal neovascularization as much as the C16Y-NP injection, which benefitted from sustained release from the polymeric NPs [50]. Injected C16Y-NPs were able to sustain therapeutic efficacy long enough to inhibit experimental neovascularization, indicating that such biodegradable polymeric DDSs may be viable alternatives for the delivery of peptide therapeutics as a treatment for choroidal neovascularization, with possibilities for other ocular/retinal illnesses.

In recent years, researchers have investigated mucoadhesive buccal drug delivery in order to overcome first-pass metabolism barriers. Buccal films avoid first-pass metabolism by taking advantage of absorption through the venous system in the cheek area [51]. Buccal drug delivery approaches have been shown to be applicable to both local [51] and systemic [52] drugs in previous studies. However, bioavailability via the buccal mucosa is relatively poor, particularly for peptides and other macromolecules, due to large molecular size, and multiple physical and chemical barriers [53]. Giovino et al., demonstrated the design of a novel hybrid buccal DDS consisting of insulin-encapsulated PEG-PLA NPs within a chitosan mucoadhesive film, designed to overcome buccal macromolecule delivery limitations and poor bioavailability. In vitro studies which looked at the release of the peptide drug (insulin) from Chitosan-films-NPs showed prolonged drug release. An initial release phase resulted in $51 \pm 8 \%$ of total peptide release in 7 
hrs, while a secondary, progressive phase resulted in a cumulative release of $70 \%$ after 15 days [53]. The results suggested that the hybrid system of NPs entrapped within a polymeric film efficiently control the release of the macromolecular peptide drug over a prolonged period of time. The use of Ch-films-NPs resulted in a 1.8 times enhancement of ex vivo insulin permeation, when compared to pure insulin alone, due to release of insulin from chitosan film and subsequently from erosion of PEG-PLA NPs [53]. This hybrid system allowed for longer residence times, overcoming rapid clearance and diffusion, and yielded higher concentrations of the macromolecule peptide drug across the buccal membrane.

\subsection{Protein Drug Delivery Systems}

Proteins are larger than peptides further limiting their abilities to permeate across physical and chemical barriers. Protein based therapeutics has been explored primarily for the treatment of type II diabetes, anti-cancer, haemophilia A and B, and thrombosis, among other diseases [54, 55]. Hu et al., reported PEG-PLA NPs conjugated with lactoferrin, a cationic iron-binding glycoprotein resulting in a DDS capable of overcoming the blood-brain barrier (BBB). The BBB effectively disallows the permeation of more than $98 \%$ of small-molecule drugs and almost $100 \%$ of macromolecular drugs [56]. Lactoferrin is a protein having many physiological roles including anti-inflammatory, immunomodulatory, anti-microbial, and anti-carcinogenic activity $[57,58]$. Furthermore, the receptors for lactoferrin protein are heavily expressed on the bloodbrain barrier facilitating its transport across. In vitro studies showed that the uptake of lactoferrin-NP conjugates was 1.45 times higher than control NPs alone [59], depicting first order kinetics dependent on time, temperature, and concentration. When evaluated in vivo in mouse models, coumarin-6, a fluorescent probe was used to evaluate brain uptake of lactoferrinNP conjugates a compared to the control NPs. Though the uptake of coumarin-6 was similar in both lactoferrin-NP and NP groups, the area under the drug concentration-time curve (AUC) and peak drug concentration $\left(\mathrm{C}_{\max }\right)$ were 2.98 and 2.86 times higher, respectively, in coumarin-6 associated with lactoferrin-NP than NP controls [59]. The evidence indicated that PEG-PLA NPs delivered protein therapeutics in a sustained, first-order release profile, and the incorporation of targeting proteins like lactoferrin, facilitated transport across BBB and internalization into target cells. Lactoferrin/PEG-PLA NP conjugates show the potential for an efficient, biocompatible, 
and non-toxic drug delivery system to the brain, particularly for macromolecules such as peptides, genes, and other proteins.

In addition, PEG-PLA NPs have also been investigated as protein carriers for nasal administration. Tobio et al., encapsulated a model protein drug, tetanus toxoid (TT) within hydrophobic PLA and surface hydrophilic PEG-PLA NPs. In vitro studies indicated that PEGPLA NPs provided a prolonged release of TT than PLA NPs [60], hypothesized to be due to PEG chain orientations towards the external aqueous mediums and the internal aqueous phase, creating a steric barrier which stabilizes the protein drug encapsulated within. In in vivo experiments, radiolabeled TT-loaded NPs were administered intra-nasaly and the amount was determined as recovered from blood circulation. One hour after administration the percentage of radioactivity recovered in blood circulation of TT-PEG-PLA NPs was 10 times that of TT-PLA NPs [60]. Biodistribution was determined by detecting radioactivity within the lymph nodes, lungs, liver, and spleen 24 hrs. post-nasal administration. The radioactivity recovered from TTPEG-PLA NPs was 3 to 6 fold higher than that of TT-PLA NPs. Control (TT aqueous solution) values were significantly lower than TT-PEG-PLA NPs and similar to that of TT-PLA NPs. The transport of the radiolabeled protein drug TT was dependent on the surface properties of the NPs, with PEG-PLA NPs providing a significantly greater penetration of TT protein drug into the circulation and lymph nodes than PLA-NPs for at least 48hrs, showing their ability for controlled, sustained protein drug release, after facilitating permeation across the nasal mucosa [60-62].

Polymeric hydrogels have been researched extensively for various therapeutic and regenerative applications [63-66]. High water content of hydrogels makes them suitable for protein delivery. Self-assembly through hydrophobic interactions of PEG-based block copolymers allows for the in situ formation of physically crosslinked hydrogels. The in situ forming hydrogels allow for the easy and homogenous loading of proteins, which is of particular interest due to the various challenges involved with protein loading and delivery. Hiemstra et al., investigated the use of biodegradable, in situ forming PEG-PLA hydrogels for the delivery of recombinant human IL-2 (rhIL-2), a therapeutic protein with proven anti-tumor activity [67]. When injected intratumorally, rhIL-2 is an extremely effective therapy against cancer [68]. In clinical trials, $63 \%$ of 
the patients with advanced nasopharyngeal carcinoma were tumor-free after five years of rHIL-2 therapy, whereas in contrast only $8 \%$ of patients who received radiotherapy were tumor-free. In vitro drug release profile of rhIL-2 from in situ forming PEG-PLA hydrogels showed nearly zero order release kinetics, with cumulative release up to $45 \%$ of loading dose in 7 days and $50 \%$ in 10 days [67]. The constant, controlled release and protection of protein therapeutic in PEG-PLA hydrogels is extremely attractive given their relatively short half-lives. Mice were injected with lymphosarcoma cells which were allowed to mature to tumors for 11 days, and then treated with rhIL-2 stereocomplexed hydrogels or free rhIL-2 in solution injected intratumorally. rhIL-2 treatments reduced tumor size and resulted in a cure rate of $30 \%$, whereas no therapeutic effects were observed when no rhIL-2 was administered [67]. The cure rate of rhIL-2 protein when delivered via PEG-PLA hydrogels was lower than that of free rhIL-2 in solution, although the difference was not statistically significant. Furthermore, therapeutic effect of rhIL-2 loaded PEGPLA hydrogels were delayed 1-2 weeks when compared to free rhIL-2. These results correlated to the slow, constant release of protein drug from the in situ forming PEG-PLA hydrogel [67]. The high water content of these hydrogels was shown to make them compatible for protein delivery, and their low viscosity allowed for easy injection, their biodegradable and biocompatible characteristics add to their attractiveness for delivery of protein therapeutics.

\subsection{Genes and Delivery Systems}

Genes are sequences or regions of the DNA varying in size from few hundred base pairs to upwards of 2 million bases that encodes or specifies the amino acid sequences of a protein. Major advances such as completion of the Human Genome Project have led to the identification and classification of genetic aberrations including diseases such as hemophilia, cystic fibrosis, muscular dystrophy, and sickle-cell anemia [69-75]. Gene therapy is based on the transfer of a proper DNA sequence to replace the disease causing gene in an affected cell with high efficiency [76]. Due to the unstable structural nature of genes, mechanisms for safe, efficient, and controllable delivery remains a limiting factor. Furthermore, unlike aptamers, they possess no particular targeting capabilities, making their use extremely risky if not combined with targeting vector molecules. The most commonly studied subset of gene therapy is for the treatment of cancer, where one approach incorporates suicide genes into malignant cells to kill them directly, 
e.g. apoptotic effector [77-79], or produce prodrug-activating enzymes [80, 81]. Another approach known a genetic correction, aims to infiltrate the infected cells and replace the diseased genes [76, 82] with a correct gene copy. Other gene delivery applications include the delivery of growth factor coding DNA sequences to treat cardiovascular [83] and neurological [84] deficiencies, as well as for regenerative medicine and wound healing [85]. Despite the superb potential of gene therapy for numerous applications their non-specific cell targeting capability is an extremely debilitating limitation. The design and use of supplementary targeting vectors (e.g. viral vectors), although having shown some success remains highly inefficient and quite inaccurate, especially considering the potentially harmful side effects of improper gene delivery [76]. Furthermore, particularly for growth factor genes, mis-targeting and variable efficiency of gene delivery leads to over-expression causing a multitude of aberrant tissue responses [76, 85].

The earliest gene therapy clinical trial was targeted to the treatment of severe combined immunodeficiency (SCID); however it was almost a decade later before some clinical success was reported by Cavazzana-Calvo et al. [86, 87]. In these early trials, the corrective genes were packaged into modified retroviruses, which have been stripped of most of their viral genes to prevent dangerous infections. However, one could control where the retroviral vectors insert themselves leading to the disruption of important genes through 'insertional mutagenesis', where disruption of a gene regulating normal cell growth and division resulted in cancer in some patients [88]. Nucleic acids such as DNA and genetic sequences have high molecular weight and a negatively charged surface, making it impermeable into the cell membrane in its native form, and furthermore exhibits instability within the ECM thereby necessitating the use of suitable delivery carriers or vectors that provide stability and ensure translocation into the cytosol and transport to the nucleus to integrate and modulate the expression of the desired protein. Various viral and non-viral vectors have demonstrated in vitro and in vivo efficacy under controlled experimental conditions, however concerns of short circulating life-span and non-specificity to the target are significant hindrances are major limitations [89-91].

Jain et al., reported using Ras-related protein Ral-A (RALA) which was complexed with DNA and cationic cell-penetrating peptides to form nanoparticles which were placed into a PLA hydrophobic core, following which the PLA core was PEGylated to form a PEG chain corona 
giving a brush like topography (Figure 4) [92]. The degradation rate of PLA defined the rate of drug delivery, while PEG corona located on the surface of the PLA core determined the biological fate of the particles. The composite nanoparticles exhibited excellent physicochemical properties including size $<200 \mathrm{~nm}$ and encapsulation efficiency $>80 \%$. Microscopy staining of the composite nanoparticles revealed the peptide-DNA nanoparticles within the PLA-PEG matrix, and by varying the properties of the PLA core the DNA release rate was modulated to be $>6$ weeks in vitro. PEGylation of particles increases their blood circulation half-life leading to enhanced bioavailability, also helping them to cross physical and physiological barriers. The PLA-PEG nanoparticles have been widely used for the safe and prolonged delivery of therapeutics owing to their versatile and defined degradation profile that can be customized according to their molecular composition and chain lengths. When the block copolymer PLAPEG is formulated into nanoparticles, PLA forms a hydrophobic core and the PEG chains form a corona around the nanoparticles giving a brush-like topography, and the degradation profile of the PLA defines the rate of drug delivery, while PEG determines the biological fate of the particles [93, 94]. All the composite nanoparticle formulations exhibited a burst release of DNA amounting to $8-0 \%$ of the therapeutic agent release in the first 24hrs. PEG chains of longer length were attributed to greater burst release due to increased contact area with water which promotes degradation rate. Formulations have a higher ratio of PLA chain length to PEG slowed the degradation profile. $\mathrm{PLA}_{25}-\mathrm{PEG}_{5}$ showed the highest amount of DNA release at every time point, which was attributed to a number of factors, longer PEG chains, shorter PLA chains, and the low $T_{\mathrm{g}}$, which was closest to the temperature of the release medium.

Targeted DNA delivery can localize treatment to a specific tissue leading to enhanced efficacy and reduced side effects, and antibodies and peptides have been used clinically for targeting [95, 96]. Peptides such as cyclic asparagines-glycine-arginine (cNGR) are stable and economical alternative to antibodies and it recognizes tumor-specific isoform of CD31 expressed specifically on vascular endothelial and perivascular cells [97]. The NGR conjugated PLA-PEG copolymers were synthesized by carbodiimide chemistry, and fabricated into NPs protecting the DNA condensed within from serum degradation largely due to the shielding effect of PEG localized on the nanoparticle surface leading to improved in vivo DNA stability (Figure 5). cNGR-PLA-PEG NPs exhibited receptor-mediated endocytosis into human umbilical vein endothelial cells 
(HUVEC) which is different from the cellular internalization pathway for untargeted nanoparticles, and contributed to significant gene transfection efficiency in comparison to controls and lipofectamine. This novel approach allowed for rapid and specific targeting to cells with upregulated CD13 thus offering potential for site-specific gene delivery.

\subsection{RNA interference (RNAi) and Therapeutics}

RNA interference using small interfering RNA (siRNA) refers to post-transcriptional gene silencing via degradation or translation inhibition of target RNA. Excellent specificity demonstrated by siRNAs allows great potential for therapeutic applications and is very commonly used to silence gene expression [98]. siRNAs are delivered as duplexes and combine to become part of a protein complex and thereby is markedly less susceptible to stability and degradation within the cell, thus exhibiting a high efficiency of targeting after cellular up-take [98] (Figure 6). For instance, the secretion of $\beta$-secretase (BACE1) is upregulated in the brains of Alzheimer patients, and this inspired siRNA-mediated knockdown of BACE1 in mouse models resulting in decreased production of various downstream proteins associated with Alzheimer's disease [99]. In another example of proposed siRNA therapeutics, the M-BCR/ABL fusion gene responsible for chronic myeloid leukemia was targeted, inducing apoptosis of the $\mathrm{BCR} / \mathrm{ABL}$ gene resulting in therapeutic equivalence to the commonly prescribed chronic myeloid leukemia drug, STI 571 [100]. Via similar knocking down mechanisms, siRNA therapeutic use has been evaluated to reduce or eliminate infections such as in HIV-1 [101, 102], Hepatitis B and C [103, 104], and influenza [105], as well as to control neurological disorders and several types of cancer [100, 106-110].

The delivery of small interfering RNA (siRNA) is a significant hurdle due to their large molecule size and polyanionic nature, which makes it difficult for these molecules to rapidly enter cells by a passive diffusion mechanism [111-113]. They remain susceptible to rapid enzymatic digestion while circulating in the blood, renal elimination, limited penetration across the capillary endothelium and inefficient cellular uptake. Yang et al. reported the fabrication of a nanoparticle delivery system composed of amphiphilic block copolymer of poly(ethylene glycol)-b-poly(d,llactide) (mPEG-PLA) along with a cationic lipid to improved siRNA encapsulation (Figure 7) 
[114]. The use of the cationic lipid $N, N$-bis(2-hydroxyethyl)- $N$-methyl- $N$-(2cholesteryloxycarbonyl aminoethyl) ammonium bromide (BHEM-Chol) ensured efficient siRNA encapsulation, and the hydrophilic PEG provided steric stabilization of the nanoparticles and protection from the physiological surroundings. The siRNA-encapsulated nanoparticles were prepared by a non-condensation process and a double-emulsion solvent evaporation technique resulting in high siRNA encapsulation efficiency. The incorporation of the cationic lipids provided nanoparticles having diameter $170-200 \mathrm{~nm}$ with a greater than $90 \%$ efficiency of siRNA encapsulation. The siRNA encapsulated nanoparticles were internalized by cancer cells resulting in significant gene knockdown and apoptosis indicating that siRNA retained integrity and released siRNA within the cancer cells. The systemic delivery of specific siRNA by nanoparticles in murine models exhibited significant inhibition and tumor suppression clearly indicating the therapeutic promise in disease treatment.

In other studies, to leverage the synergistic effects of chemotherapeutant and microRNA (miRNA) on tumor therapy Qian et al., constructed dendrimer analogs with an amphiphilic starbranched copolymers comprising PLA and polydimethylaminoethyl methacrylate (PDMAEMA) of varying molecular architectures for co-delivery of miRNA and doxorubicin [115]. Thought separate delivery systems have shown success if delivering drug alone or miRNA alone, the authors overcame the difficulty in delivering therapeutic miRNA and chemo-drugs into same tumor cells maximizing intracellular cooperation. The gene transfection efficiency and tumor inhibition ability was demonstrated to be dependent on the molecular architecture of the designed star branched copolymers $[116,117]$. Star polymers are three-dimensional polymers having multiple arms all of which are connected to a central core, and compared with linear polymers, they have very high molecular weight but still possesses characteristics of solubility and viscosity similar to that of linear or branched polymers of relatively low molecular weights. Amphiphilic branched star polymers of the $A_{3},\left(A B_{3}\right)_{2}$, and $\left(A B_{3}\right)_{3}$ architecture were synthesized using PLA as a hydrophobic branch and PDMAEMA as a hydrophilic core (Figure 8). The proton buffering effect exhibited by PDMAEMA correlated with increasing gene transfection efficiency, and thus structures with the highest degree of PDMAEMA repeat units were found to be most efficient at gene transfection. The biodegradable and hydrophobic nature of the PLA core was optimal to package and deliver doxorubicin, a hydrophobic drug. 
In other strategies, a multifunctional fluorescent nanocomposite was developed by Dong et al., demonstrating several applications including cell imaging, intracellular miRNAs regulation, and multiple gene probe delivery (Figure 9) [118-122]. A positive charged polymer of biocompatible PEG and biodegradable PLA was covalently grafted on graphene quantum dots (GQDs), and in addition, gene probes were adsorbed onto the surface of the multifunctional GQDs. Photoluminescent (PL) graphene quantum dots (GQDs) have a large surface area, superior mechanical flexibility, exhibit optical and electronic properties offering great potential in a variety of biomedical applications. The authors demonstrated uptake and monitoring of the GQDs in HeLa cells, and furthermore recognized the fluorescent signal produced by the gene probes regulating the gene target. Functionalization with PEG and PLA polymers by co-grafting ensured good biocompatibility, low cytotoxicity, and excellent physiological stability. In addition, the simultaneous loading of multiple gene-probes onto the functionalized GQDs provide new possibilities in diagnosis and treatment in parallel, which is of tremendous benefit in health care. For instance, the simultaneous conjugation of miRNA-21-targeting and survivintargeting agents on the GQDs induced better inhibition of cancer cell growth and more apoptosis

of cancer cells, compared with conjugation of agents targeting miRNA-21 or survivin alone, along with intracellular miRNA imaging analysis.

\section{Conclusions and Future Perspective}

Biomacromolecules have emerged as an important component of disease treatment especially involving genetic aberrations, immune modulation, vaccines and cancer. The size and charge of the biomolecule, its susceptibility to chemical and enzymatic degradation in the body, and challenges in target specificity are leading to innovative strategies largely utilizing synthetic polymers especially polyesters. The fabrication of delivery systems for peptides, proteins, and nucleotide molecules for controlled release is challenging, and successful drug delivery systems will incorporate biomolecule protection, stabilization, long half-life, and tissue targeting technologies, and combinations of these approaches. Emergent engineering approaches such as pegylation, peptide/PLA co-polymer synthesis, surface modification, and conjugation with cell penetrating peptides have addressed some of these challenges. 
PLA is a linear, lipophilic, biodegradable polymer composed of lactic acid monomer that is used in a number of FDA approved medical products including sutures and implants [123-125]. Lactic acid, a component of various metabolic pathways in the human body, is the only degradation product formed following PLA hydrolysis making it a biomaterial of significant interest for a variety of biomedical applications [126]. The increased competition and wide-scale changes in the biopharmaceutical industry such as targeted products, personalized medicine, and orphan diseases, have led to manufacturing trends of limited production runs and product-specific requirements. This industry-wide shift in approach demands evolution in new technologies and process methodology to improve efficiency, scalability and batch reproducibility across all manufacturing units. In addition to addressing scalability, improving process robustness, and development of single-use systems, biomanufacturing centers have fostered new partnerships as the manufacturing process, including sterilization, and packaging have become more central to the effectiveness of drugs and delivery systems. Biomolecules are extremely challenging in this regard due to their structural and functional complexity, and their dynamic nature, as imperfect processing techniques will hamper clinical translation. Exciting future opportunities lies in the

therapeutic target space, especially technologies that allow intracellular access and localized tissue-specific accumulation. The in vitro and in vivo models of characterization are very promising helping to determine a range for therapeutic efficacy and safety, however clinical trials are critical to validate the therapeutic strategy and to optimize parameters for clinical setting. Future work must address the above concerns determining the efficiencies, scalability to commercial production, and validating the technological and manufacturing innovation.

\section{Acknowledgements}

Authors acknowledge funding from the Connecticut Regenerative Medicine Research Fund-15RMB-UCHC-08 in support of this work. Authors also acknowledge the funding support from the National Science Foundation Award Numbers IIP-1311907, IIP-1355327 and EFRI-1332329. 


\section{Figure Legends}

Figure 1: Schematic illustrating the selection of nucleic acid aptamers in vitro. An initial library isolated using SELEX is presented to the surface-bound target. Given time, certain molecules bind to the target, and are selected into a separate library (Step 1). The selected library is amplified using PCR (Step 2). Successive rounds of selection further refine the library (Step 3). Iterative purification leads to an optimal nucleic acid aptamer (Step 4).

Figure 2: Screening techniques for high-affinity peptide candidates. Legend: yellow = target of interest, blue $=$ peptide of interest, grey $=$ other targets/peptides. Peptide affinity towards a target(s) of interest can be analyzed by immobilizing either the target or the peptide of interest and identifying the highest-affinity binding species. Alternatively, peptides fused to bacteriophages can bind to a target, known as phage display.

Figure 3. In vivo glioma distribution of (A) NP, (B) NP with tLyp-1, (C) F3-NP and (D) F3-NP with tLyp-1 $3 \mathrm{~h}$ after tail vein injection. Red lines showed the boundary between glioma and normal brain tissue. Arrows indicated the glioma zones. Bar: $100 \mu \mathrm{m}$. [49]

Figure 4. Sequence and structure of RALA. Overview of composition of the composite nanoparticles. [92]

Notes: (A) Primary sequence (top), secondary structure (middle), and surface representations of the RALA peptide, bottom left shows rotation to reveal the hydrophobic side, bottom right shows rotation to reveal the hydrophilic side. (B) Schematic representation of polymeric-cationic peptide composite nanoparticles.

Abbreviations: pDNA, plasmid DNA; PEG, polyethylene glycol; PLA, polylactic acid; RNPs, RALA nanoparticles.

Figure 5. Schematic route of the preparation of cNGR-PLA-PEG NPs. [97]

Figure 6: Mechanism of siRNA gene silencing. siRNA duplex is taken up by Argonaute (AGO) protein, where only a single (guide) strand is retained as part of the RNA-induced silencing complex (RISC). The siRNA guide strand guides the RISC to the target mRNA, determined by 
complete base pair matching. The binding of the RISC to the target mRNA induces cleavage of the mRNA strand, which is later degraded.

Figure 7. Schematic illustration of the fabrication of cationic lipid assisted nanoparticles with siRNA encapsulation. mPEG-PLA: poly(ethylene glycol)-b-poly(d,l-lactide); BHEM-Chol: N,Nbis(2-hydroxyethyl)-N-methyl-N-(2-cholesteryloxycarbonyl aminoethyl) ammonium bromide; W and O represent water phase and oil phase, respectively. [114]

Figure 8. Illustration of co-delivery of DOX and miR-21i by star-branched amphiphilic copolymer micelles and the mechanism for enhancement of chemosensitivity of glioma cells. [115]

Figure 9. Schematic presentation of f-GQDs-based cell imaging and combined specific genetargeting agents delivery. Probe 1 is the inhibitor probe of miRNA-21, and probe 2 is survivin antisense oligodeoxynucleotide. [118] 
Figure 1

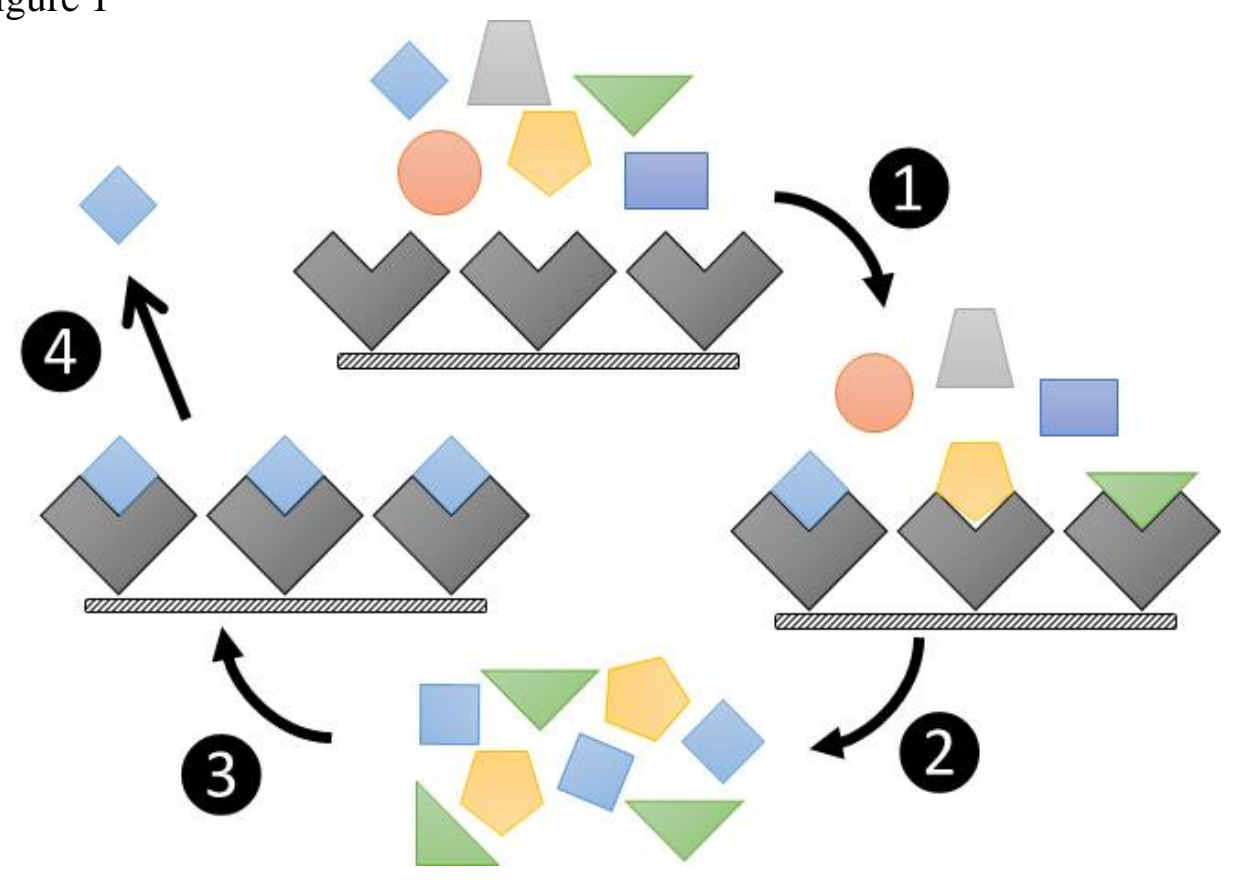

Figure 2

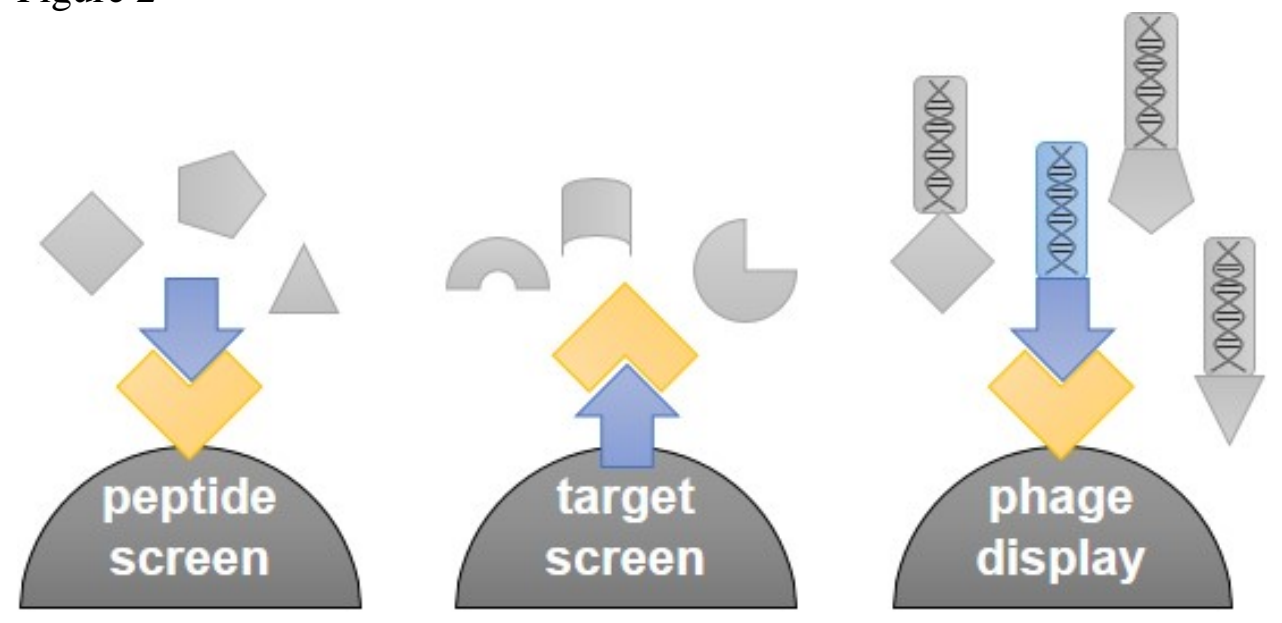


Figure 3

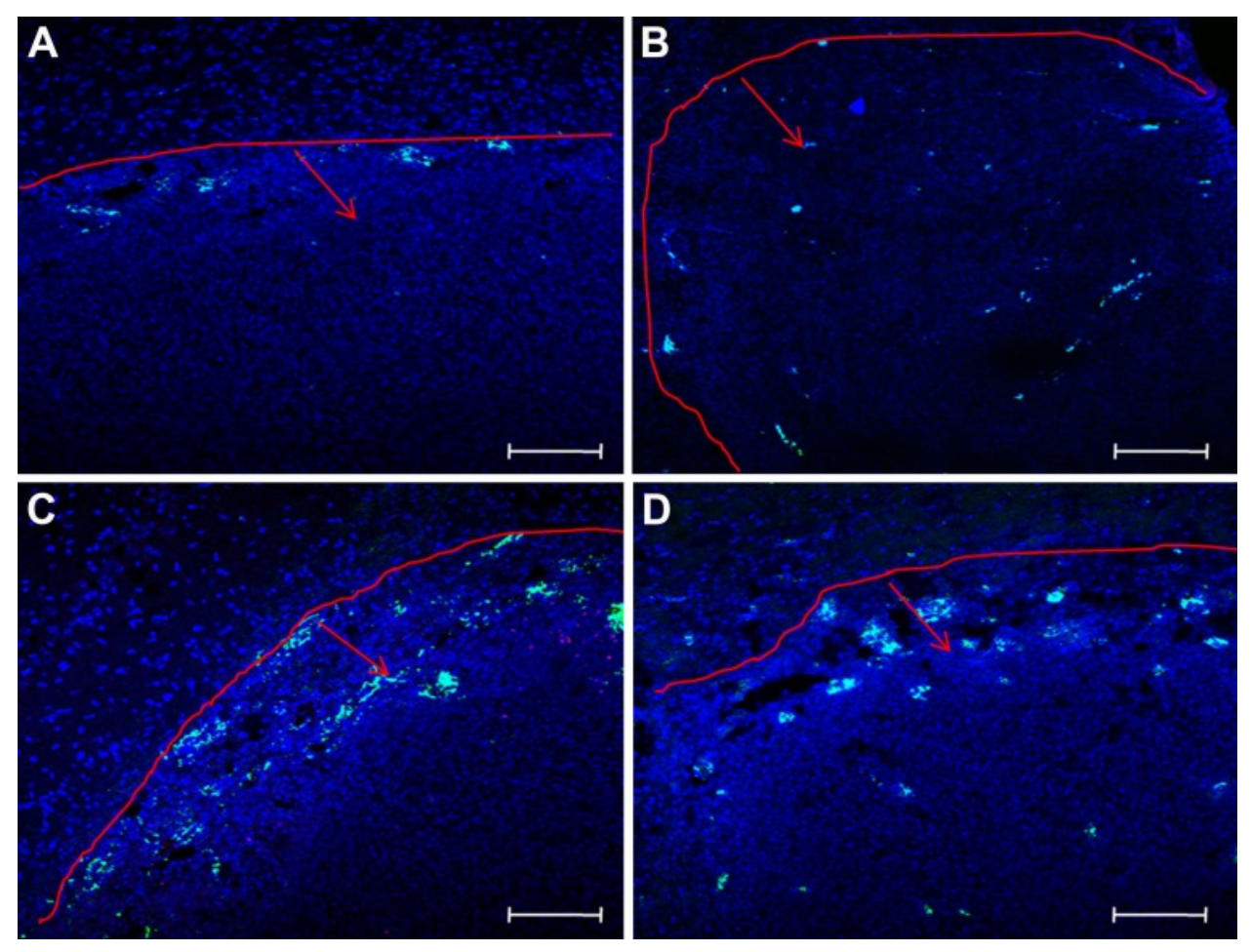


Figure 4
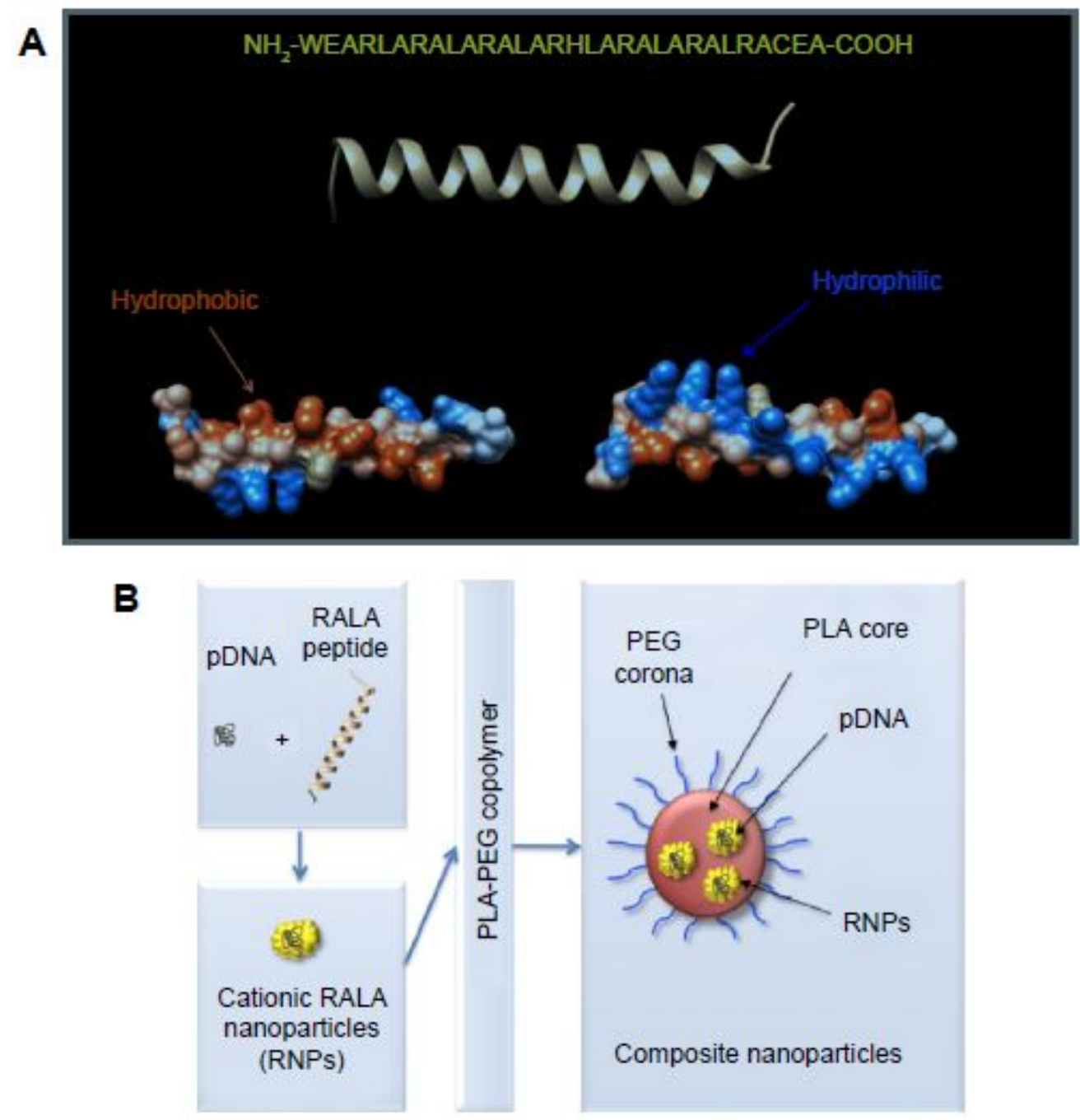
Figure 5

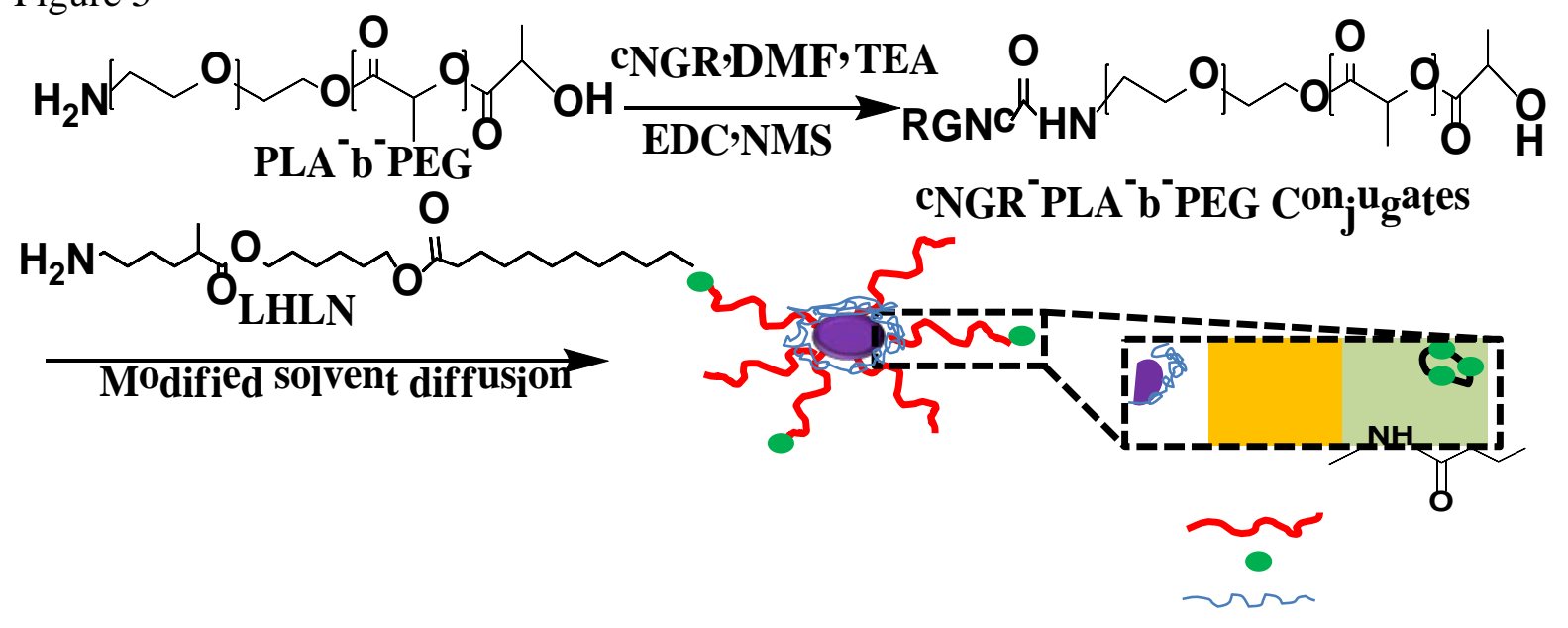

Figure 6
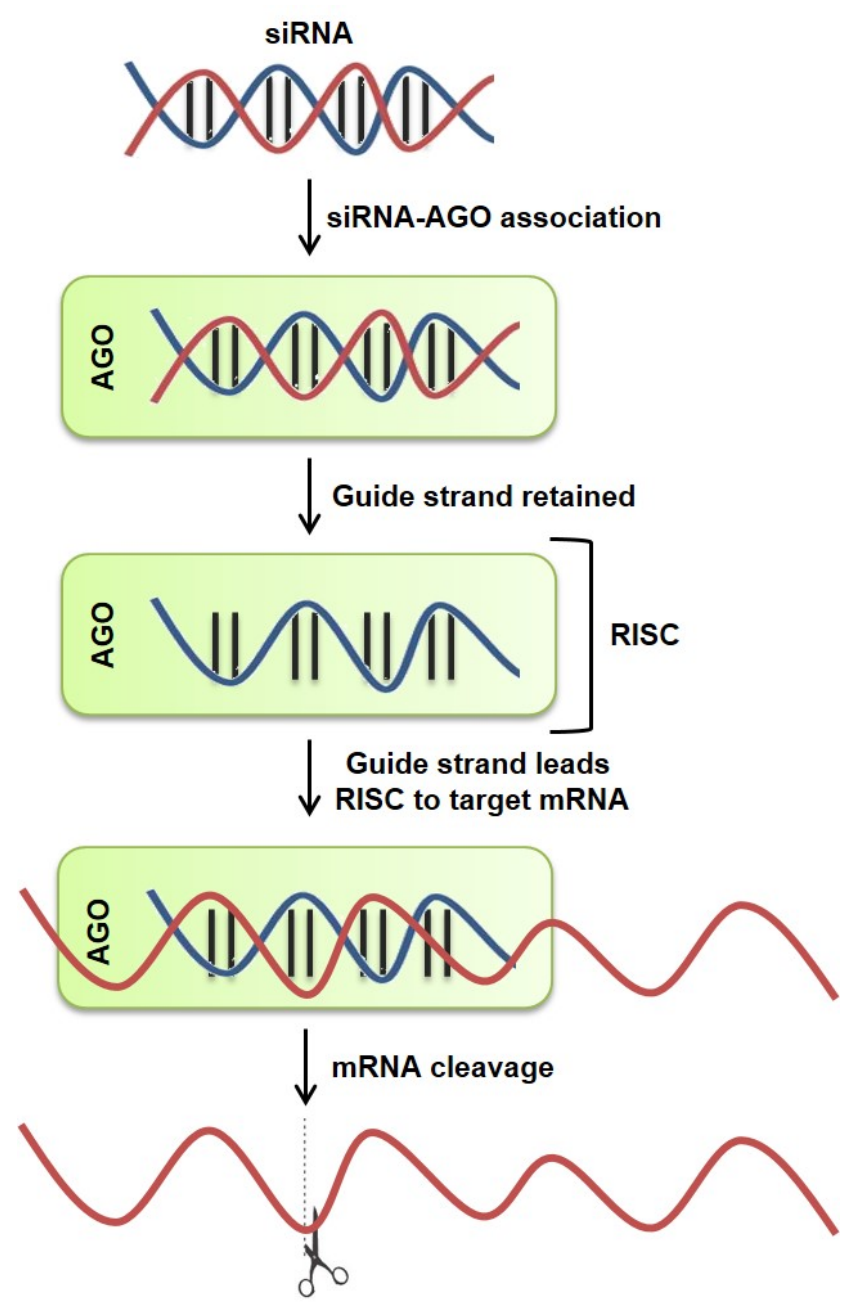
Figure 7

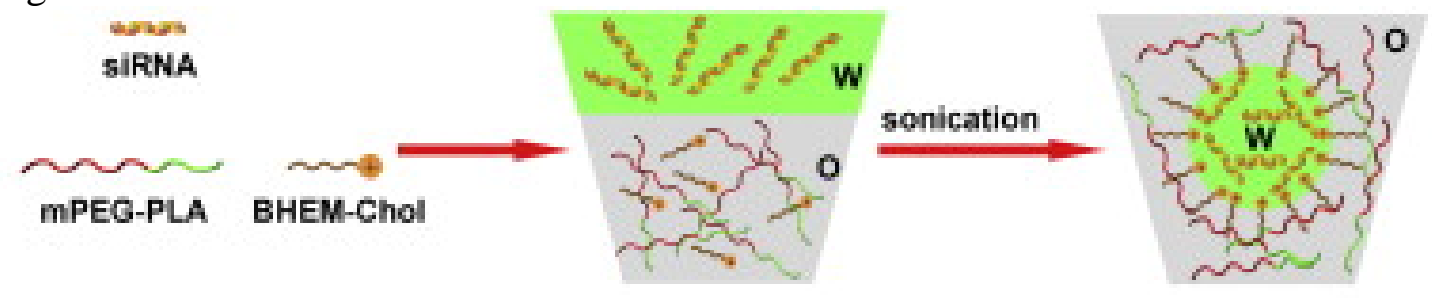

Primary emulsion
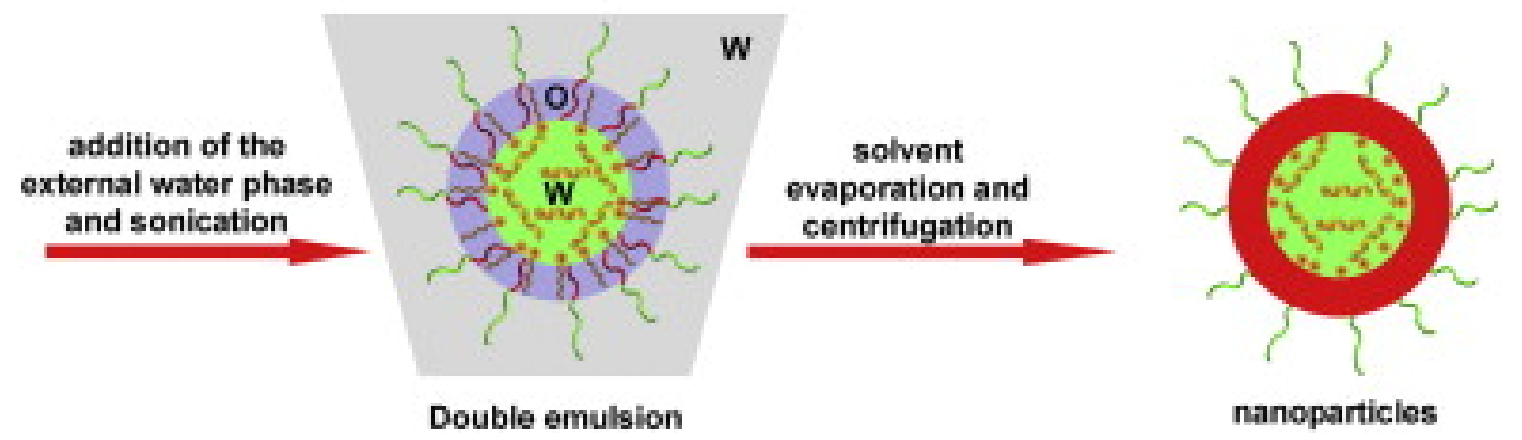

Figure 8

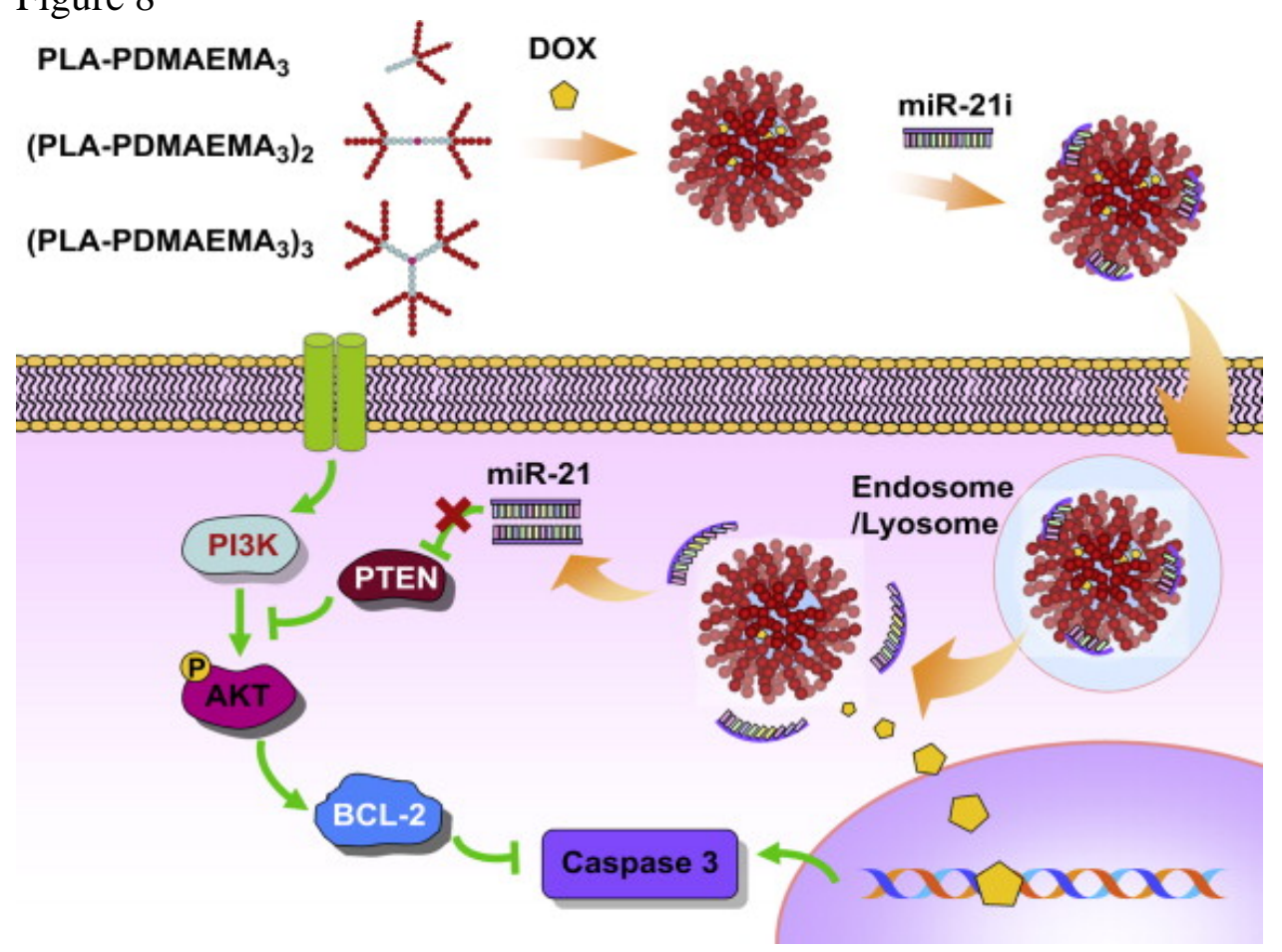


Figure 9

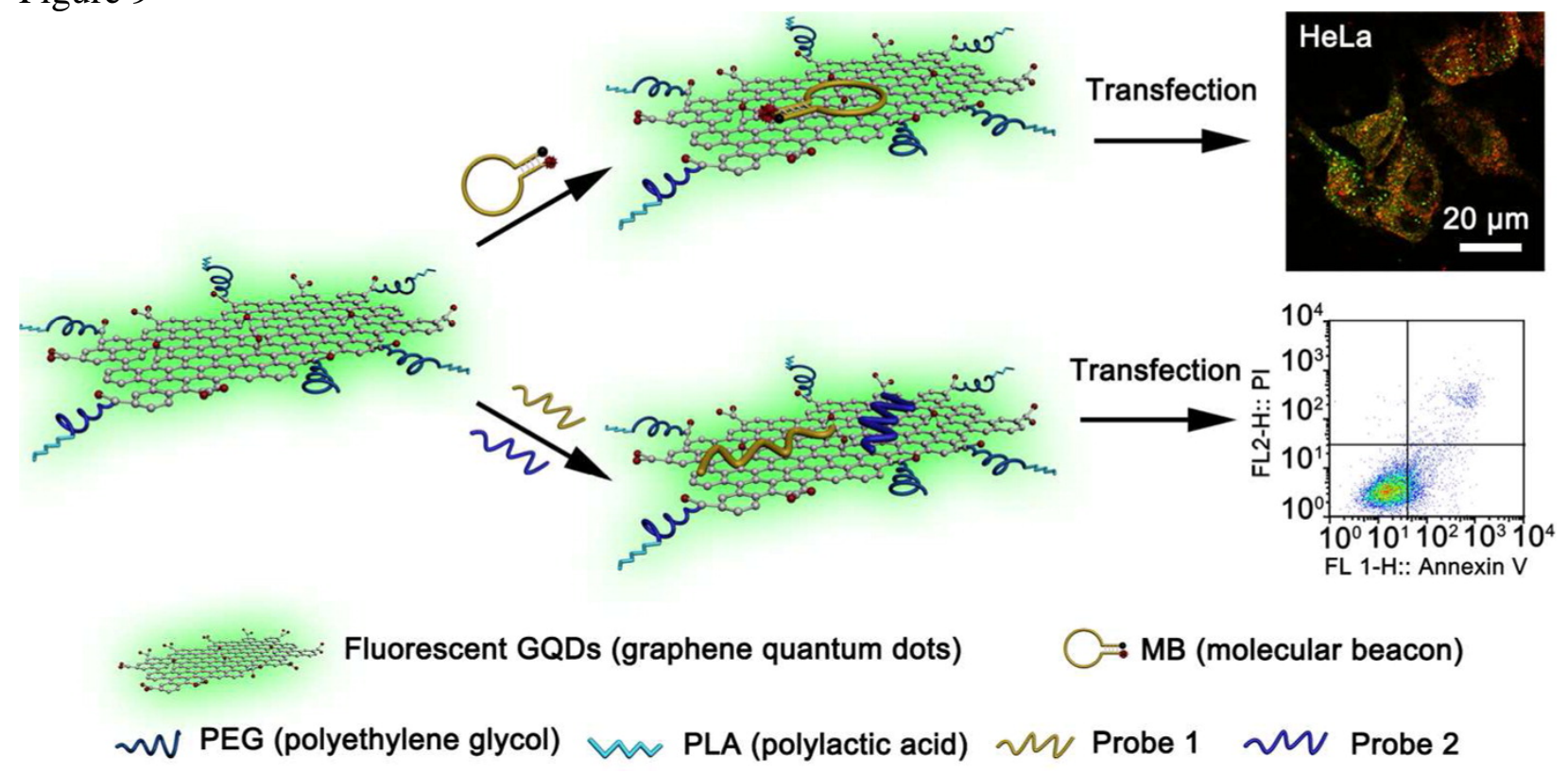




\section{References}

[1] M.M.-C. Cheng, G. Cuda, Y.L. Bunimovich, M. Gaspari, J.R. Heath, H.D. Hill, C.A. Mirkin, A.J. Nijdam, R. Terracciano, T. Thundat, M. Ferrari, Nanotechnologies for biomolecular detection and medical diagnostics, Current Opinion in Chemical Biology, 10 (2006) 11-19.

[2] T. Vo-Dinh, B.M. Cullum, D.L. Stokes, Nanosensors and biochips: frontiers in biomolecular diagnostics, Sensors and Actuators B: Chemical, 74 (2001) 2-11.

[3] J. Bergstrom, S. Lofas, B. Johnsson, Sensing surfaces capable of selective biomolecular interactions, to be used in biosensor systems, in, Google Patents, 1993.

[4] S.K. Sharma, N. Sehgal, A. Kumar, Biomolecules for development of biosensors and their applications, Current Applied Physics, 3 (2003) 307-316.

[5] J.J.T. Santini, A.C. Richards, R. Scheidt, M.J. Cima, R. Langer, Microchips as Controlled Drug-Delivery Devices, Angewandte Chemie International Edition, 39 (2000) 2396-2407.

[6] G. Ma, Microencapsulation of protein drugs for drug delivery: Strategy, preparation, and applications, Journal of Controlled Release, 193 (2014) 324-340.

[7] S.H. Hyon, Biodegradable poly (lactic acid) microspheres for drug delivery systems, Yonsei medical journal, 41 (2000) 720-734.

[8] R. Jain, N.H. Shah, A.W. Malick, C.T. Rhodes, Controlled drug delivery by biodegradable poly(ester) devices: different preparative approaches, Drug development and industrial pharmacy, 24 (1998) 703-727.

[9] V. Lassalle, M.L. Ferreira, PLA nano- and microparticles for drug delivery: an overview of the methods of preparation, Macromol Biosci, 7 (2007) 767-783.

[10] H. Okada, Drug discovery by formulation design and innovative drug delivery systems (DDS), Yakugaku zasshi : Journal of the Pharmaceutical Society of Japan, 131 (2011) 12711287.

[11] E.M. Saffer, G.N. Tew, S.R. Bhatia, Poly(lactic acid)-poly(ethylene oxide) block copolymers: new directions in self-assembly and biomedical applications, Current medicinal chemistry, 18 (2011) 5676-5686.

[12] N. Saito, N. Murakami, J. Takahashi, H. Horiuchi, H. Ota, H. Kato, T. Okada, K. Nozaki, K. Takaoka, Synthetic biodegradable polymers as drug delivery systems for bone morphogenetic proteins, Advanced drug delivery reviews, 57 (2005) 1037-1048. 
[13] J.K. Vasir, V. Labhasetwar, Biodegradable nanoparticles for cytosolic delivery of therapeutics, Advanced drug delivery reviews, 59 (2007) 718-728.

[14] M.R. Vijayakumar, M.S. Muthu, S. Singh, Copolymers of poly(lactic acid) and D-alphatocopheryl polyethylene glycol 1000 succinate-based nanomedicines: versatile multifunctional platforms for cancer diagnosis and therapy, Expert opinion on drug delivery, 10 (2013) 529-543.

[15] K. Avgoustakis, Pegylated poly(lactide) and poly(lactide-co-glycolide) nanoparticles: preparation, properties and possible applications in drug delivery, Current drug delivery, 1 (2004) 321-333.

[16] H. Tsuji, Poly(lactide) stereocomplexes: formation, structure, properties, degradation, and applications, Macromol Biosci, 5 (2005) 569-597.

[17] D.S. Pisal, M.P. Kosloski, S.V. Balu-Iyer, DELIVERY OF THERAPEUTIC PROTEINS, Journal of pharmaceutical sciences, 99 (2010) 2557-2575.

[18] M. Hacker, W.S. Messer II, K.A. Bachmann, Pharmacology: principles and practice, Academic Press, 2009.

[19] J.M. Healy, S.D. Lewis, M. Kurz, R.M. Boomer, K.M. Thompson, C. Wilson, T.G. McCauley, Pharmacokinetics and biodistribution of novel aptamer compositions, Pharmaceutical research, 21 (2004) 2234-2246.

[20] R.S. Kadam, D.W. Bourne, U.B. Kompella, Nano-advantage in enhanced drug delivery with biodegradable nanoparticles: contribution of reduced clearance, Drug metabolism and disposition: the biological fate of chemicals, 40 (2012) 1380-1388.

[21] J.F. Coelho, P.C. Ferreira, P. Alves, R. Cordeiro, A.C. Fonseca, J.R. Góis, M.H. Gil, Drug delivery systems: Advanced technologies potentially applicable in personalized treatments, The EPMA Journal, 1 (2010) 164-209.

[22] T.S. Levchenko, R. Rammohan, A.N. Lukyanov, K.R. Whiteman, V.P. Torchilin, Liposome clearance in mice: the effect of a separate and combined presence of surface charge and polymer coating, International journal of pharmaceutics, 240 (2002) 95-102.

[23] K. Gavrilov, W.M. Saltzman, Therapeutic siRNA: Principles, Challenges, and Strategies, The Yale Journal of Biology and Medicine, 85 (2012) 187-200.

[24] R. Juliano, A. Astriab-Fisher, D. Falke, Macromolecular therapeutics, Molecular interventions, 1 (2001) 40. 
[25] T. Lehto, K. Kurrikoff, Ü. Langel, Cell-penetrating peptides for the delivery of nucleic acids, Expert opinion on drug delivery, 9 (2012) 823-836.

[26] B. Gupta, T.S. Levchenko, V.P. Torchilin, Intracellular delivery of large molecules and small particles by cell-penetrating proteins and peptides, Advanced drug delivery reviews, 57 (2005) 637-651.

[27] M. Hallbrink, A. Floren, A. Elmquist, M. Pooga, T. Bartfai, U. Langel, Cargo delivery kinetics of cell-penetrating peptides, Biochimica et biophysica acta, 1515 (2001) 101-109.

[28] G.P. Dietz, M. Bahr, Delivery of bioactive molecules into the cell: the Trojan horse approach, Molecular and cellular neurosciences, 27 (2004) 85-131.

[29] M. Mae, U. Langel, Cell-penetrating peptides as vectors for peptide, protein and oligonucleotide delivery, Current opinion in pharmacology, 6 (2006) 509-514.

[30] T. Lehto, K. Kurrikoff, U. Langel, Cell-penetrating peptides for the delivery of nucleic acids, Expert opinion on drug delivery, 9 (2012) 823-836.

[31] P. Jarver, K. Langel, S. El-Andaloussi, U. Langel, Applications of cell-penetrating peptides in regulation of gene expression, Biochem Soc Trans, 35 (2007) 770-774.

[32] B.R. Meade, S.F. Dowdy, Enhancing the cellular uptake of siRNA duplexes following noncovalent packaging with protein transduction domain peptides, Advanced drug delivery reviews, 60 (2008) 530-536.

[33] P. Järver, K. Langel, S. El-Andaloussi, Ü. Langel, Applications of cell-penetrating peptides in regulation of gene expression, Biochemical Society Transactions, 35 (2007) 770-774.

[34] H. Xia, X. Gao, G. Gu, Z. Liu, Q. Hu, Y. Tu, Q. Song, L. Yao, Z. Pang, X. Jiang, J. Chen, H. Chen, Penetratin-functionalized PEG-PLA nanoparticles for brain drug delivery, International journal of pharmaceutics, 436 (2012) 840-850.

[35] T. Saga, R.D. Neumann, T. Heya, J. Sato, S. Kinuya, N. Le, C.H. Paik, J.N. Weinstein, Targeting cancer micrometastases with monoclonal antibodies: a binding-site barrier, Proceedings of the National Academy of Sciences, 92 (1995) 8999-9003.

[36] T.W. Speer, Targeted radionuclide therapy, Lippincott Williams \& Wilkins, 2012.

[37] R. Solaro, F. Chiellini, A. Battisti, Targeted delivery of protein drugs by nanocarriers, Materials, 3 (2010) 1928-1980.

[38] L.M. Bareford, P.W. Swaan, ENDOCYTIC MECHANISMS FOR TARGETED DRUG DELIVERY, Advanced drug delivery reviews, 59 (2007) 748-758. 
[39] I. Nakase, S. Kobayashi, S. Futaki, Endosome-disruptive peptides for improving cytosolic delivery of bioactive macromolecules, Peptide Science, 94 (2010) 763-770.

[40] E. Wagner, Application of membrane-active peptides for nonviral gene delivery, Advanced drug delivery reviews, 38 (1999) 279-289.

[41] R.R. White, B.A. Sullenger, C.P. Rusconi, Developing aptamers into therapeutics, The Journal of clinical investigation, 106 (2000) 929-934.

[42] T. Uhlig, T. Kyprianou, F.G. Martinelli, C.A. Oppici, D. Heiligers, D. Hills, X.R. Calvo, P. Verhaert, The emergence of peptides in the pharmaceutical business: From exploration to exploitation, EuPA Open Proteomics, 4 (2014) 58-69.

[43] J.L. Fox, Antimicrobial peptides stage a comeback, Nature biotechnology, 31 (2013) 379382.

[44] Z. Zeng, C.-H. Tung, Y. Zu, A Cancer Cell-Activatable Aptamer-Reporter System for OneStep Assay of Circulating Tumor Cells, Mol Ther Nucleic Acids, 3 (2014) e184.

[45] P. Sundaram, H. Kurniawan, M.E. Byrne, J. Wower, Therapeutic RNA aptamers in clinical trials, European Journal of Pharmaceutical Sciences, 48 (2013) 259-271.

[46] M.C. Veronesi, Y. Aldouby, A.J. Domb, M.J. Kubek, Thyrotropin-releasing hormone d, l polylactide nanoparticles (TRH-NPs) protect against glutamate toxicity in vitro and kindling development in vivo, Brain research, 1303 (2009) 151-160.

[47] P.Y. Wen, S. Kesari, Malignant gliomas in adults, New England Journal of Medicine, 359 (2008) 492-507.

[48] A. Behin, K. Hoang-Xuan, A.F. Carpentier, J.-Y. Delattre, Primary brain tumours in adults, The Lancet, 361 (2003) 323-331.

[49] Q. Hu, G. Gu, Z. Liu, M. Jiang, T. Kang, D. Miao, Y. Tu, Z. Pang, Q. Song, L. Yao, F3 peptide-functionalized PEG-PLA nanoparticles co-administrated with tLyp-1 peptide for antiglioma drug delivery, Biomaterials, 34 (2013) 1135-1145.

[50] H. Kim, K.G. Csaky, Nanoparticle-integrin antagonist C16Y peptide treatment of choroidal neovascularization in rats, Journal of Controlled Release, 142 (2010) 286-293.

[51] C.A. Squier, P.W. Wertz, Structure and function of the oral mucosa and implications for drug delivery, Drugs and the pharmaceutical sciences, 74 (1996) 1-26.

[52] J.A. Hoogstraate, P.W. Wertz, Drug delivery via the buccal mucosa, Pharmaceutical Science \& Technology Today, 1 (1998) 309-316. 
[53] C. Giovino, I. Ayensu, J. Tetteh, J.S. Boateng, An integrated buccal delivery system combining chitosan films impregnated with peptide loaded PEG-b-PLA nanoparticles, Colloids and Surfaces B: Biointerfaces, 112 (2013) 9-15.

[54] C. Sarisozen, V.P. Torchilin, Intracellular Delivery of Proteins and Peptides, Drug Delivery: Principles and Applications, Second Edition, (2016) 576-622.

[55] M. Manco-Johnson, L. Bomgaars, J. Palascak, A. Shapiro, J. Geil, S. Fritsch, B. Pavlova, D. Gelmont, Efficacy and safety of protein C concentrate to treat purpura fulminans and thromboembolic events in severe congenital protein C deficiency, Thrombosis and Haemostasis, (2016).

[56] W.M. Pardridge, Drug targeting to the brain, Pharmaceutical research, 24 (2007) 17331744.

[57] A. Jhaveri, S.S. Arya, Lactoferrin: health benefits, technology and applications, AGRO FOOD INDUSTRY HI-TECH, 26 (2015) 40-43.

[58] S. Mayeur, S. Spahis, Y. Pouliot, E. Levy, Lactoferrin, a Pleiotropic Protein in Health and Disease, Antioxidants \& Redox Signaling, (2016).

[59] K. Hu, J. Li, Y. Shen, W. Lu, X. Gao, Q. Zhang, X. Jiang, Lactoferrin-conjugated PEGPLA nanoparticles with improved brain delivery: in vitro and in vivo evaluations, Journal of controlled release, 134 (2009) 55-61.

[60] M. Tobio, R. Gref, A. Sanchez, R. Langer, M. Alonso, Stealth PLA-PEG nanoparticles as protein carriers for nasal administration, Pharmaceutical research, 15 (1998) 270-275.

[61] C.Y. Lin, S.J. Lin, Y.C. Yang, D.Y. Wang, H.F. Cheng, M.K. Yeh, Biodegradable polymeric microsphere-based vaccines and their applications in infectious diseases, Human vaccines \& immunotherapeutics, 11 (2015) 650-656.

[62] H. Soni, Formulation evaluation and in vitro and in vivo studies of PLA based vaccine for tuberculosis, (2015).

[63] H. Mao, G. Shan, Y. Bao, Z.L. Wu, P. Pan, Thermoresponsive physical hydrogels of poly (lactic acid)/poly (ethylene glycol) stereoblock copolymers tuned by stereostructure and sequence of hydrophobic blocks, Soft Matter, (2016).

[64] N. Peppas, P. Bures, W. Leobandung, H. Ichikawa, Hydrogels in pharmaceutical formulations, European journal of pharmaceutics and biopharmaceutics, 50 (2000) 27-46. 
[65] B. Jeong, Y.H. Bae, D.S. Lee, S.W. Kim, Biodegradable block copolymers as injectable drug-delivery systems, Nature, 388 (1997) 860-862.

[66] D. Chitkara, A. Shikanov, N. Kumar, A.J. Domb, Biodegradable injectable in situ depotforming drug delivery systems, Macromolecular bioscience, 6 (2006) 977-990.

[67] C. Hiemstra, Z. Zhong, S.R. Van Tomme, M.J. van Steenbergen, J.J. Jacobs, W. Den Otter, W.E. Hennink, J. Feijen, In vitro and in vivo protein delivery from in situ forming poly (ethylene glycol)-poly (lactide) hydrogels, Journal of controlled release, 119 (2007) 320-327.

[68] J.J. Jacobs, G.J. Hordijk, I.M. Jürgenliemk-Schulz, C.H. Terhaard, J.W. Koten, J.J. Battermann, W. Den Otter, Treatment of stage III-IV nasopharyngeal carcinomas by external beam irradiation and local low doses of IL-2, Cancer Immunology, Immunotherapy, 54 (2005) 792-798.

[69] C.E. Walsh, Gene therapy progress and prospects: gene therapy for the hemophilias, Gene therapy, 10 (2003) 999-1003.

[70] S. Ferrari, D.M. Geddes, E.W. Alton, Barriers to and new approaches for gene therapy and gene delivery in cystic fibrosis, Advanced drug delivery reviews, 54 (2002) 1373-1393.

[71] J.C.T. van Deutekom, G.-J.B. van Ommen, Advances in Duchenne muscular dystrophy gene therapy, Nat Rev Genet, 4 (2003) 774-783.

[72] D. Wang, G. Gao, State-of-the-art human gene therapy: part I. Gene delivery technologies, Discovery medicine, 18 (2014) 67-77.

[73] D. Wang, G. Gao, State-of-the-art human gene therapy: part II. Gene therapy strategies and clinical applications, Discovery medicine, 18 (2014) 151-161.

[74] Y.Y. Jang, L. Cai, Z. Ye, Genome editing systems in novel therapies, Discovery medicine, 21 (2016) 57-64.

[75] K. Papadopoulos, P. Wattanaarsakit, W. Prasongchean, R. Narain, Gene therapies in clinical trials, Polymers and Nanomaterials for Gene Therapy, (2016) 231.

[76] D.W. Pack, A.S. Hoffman, S. Pun, P.S. Stayton, Design and development of polymers for gene delivery, Nature Reviews Drug Discovery, 4 (2005) 581-593.

[77] F. Moolten, Drug sensitivity (" suicide") genes for selective cancer chemotherapy, Cancer gene therapy, 1 (1994) 279-287.

[78] D.W. Nicholson, From bench to clinic with apoptosis-based therapeutic agents, Nature, 407 (2000) 810-816. 
[79] J.C. Reed, Apoptosis-targeted therapies for cancer, Cancer Cell, 3 (2003) 17-22.

[80] M. Aghi, F. Hochberg, X.O. Breakefield, Prodrug activation enzymes in cancer gene therapy, The journal of gene medicine, 2 (2000) 148-164.

[81] C.J. Springer, I. Niculescu-Duvaz, Prodrug-activating systems in suicide gene therapy, Journal of Clinical Investigation, 105 (2000) 1161-1167.

[82] R. Vile, S. Russell, N. Lemoine, Cancer gene therapy: hard lessons and new courses, Gene therapy, 7 (2000) 2-8.

[83] V.J. Dzau, K. Beatt, G. Pompilio, K. Smith, Current perceptions of cardiovascular gene therapy, The American journal of cardiology, 92 (2003) 18-23.

[84] M.H. Tuszynski, Growth-factor gene therapy for neurodegenerative disorders, The Lancet Neurology, 1 (2002) 51-57.

[85] K.R. Cutroneo, Gene therapy for tissue regeneration, Journal of cellular biochemistry, 88 (2003) 418-425.

[86] M. Cavazzana-Calvo, S. Hacein-Bey, G. de Saint Basile, F. Gross, E. Yvon, P. Nusbaum, F. Selz, C. Hue, S. Certain, J.L. Casanova, P. Bousso, F.L. Deist, A. Fischer, Gene therapy of human severe combined immunodeficiency (SCID)-X1 disease, Science (New York, N.Y.), 288 (2000) 669-672.

[87] I.M. Verma, N. Somia, Gene therapy -- promises, problems and prospects, Nature, 389 (1997) 239-242.

[88] E. Check, Gene therapy: A tragic setback, Nature, 420 (2002) 116-118.

[89] T.G. Park, J.H. Jeong, S.W. Kim, Current status of polymeric gene delivery systems, Advanced drug delivery reviews, 58 (2006) 467-486.

[90] E. Koren, V.P. Torchilin, Cell-penetrating peptides: breaking through to the other side, Trends in molecular medicine, 18 (2012) 385-393.

[91] S. Deshayes, M.C. Morris, G. Divita, F. Heitz, Cell-penetrating peptides: tools for intracellular delivery of therapeutics, Cellular and molecular life sciences : CMLS, 62 (2005) 1839-1849.

[92] A.K. Jain, A. Massey, H. Yusuf, D.M. McDonald, H.O. McCarthy, V.L. Kett, Development of polymeric-cationic peptide composite nanoparticles, a nanoparticle-in-nanoparticle system for controlled gene delivery, International journal of nanomedicine, 10 (2015) 7183-7196. 
[93] R. Gref, A. Domb, P. Quellec, T. Blunk, R.H. Muller, J.M. Verbavatz, R. Langer, The controlled intravenous delivery of drugs using PEG-coated sterically stabilized nanospheres, Advanced drug delivery reviews, 16 (1995) 215-233.

[94] J. Suh, K.L. Choy, S.K. Lai, J.S. Suk, B.C. Tang, S. Prabhu, J. Hanes, PEGylation of nanoparticles improves their cytoplasmic transport, International journal of nanomedicine, 2 (2007) 735-741.

[95] Z. Hu, F. Luo, Y. Pan, C. Hou, L. Ren, J. Chen, J. Wang, Y. Zhang, Arg-Gly-Asp (RGD) peptide conjugated poly (lactic acid)-poly (ethylene oxide) micelle for targeted drug delivery, Journal of Biomedical Materials Research Part A, 85 (2008) 797-807.

[96] Z. Liu, X. Gao, T. Kang, M. Jiang, D. Miao, G. Gu, Q. Hu, Q. Song, L. Yao, Y. Tu, B6 peptide-modified PEG-PLA nanoparticles for enhanced brain delivery of neuroprotective peptide, Bioconjugate chemistry, 24 (2013) 997-1007.

[97] C. Liu, W. Yu, Z. Chen, J. Zhang, N. Zhang, cNGR conjugated poly(lactic acid)poly(ethylene glycol) nanoparticles for targeted gene delivery, Journal of Controlled Release, 152, Supplement 1 (2011) e155-e157.

[98] J.K. Watts, D.R. Corey, Gene silencing by siRNAs and antisense oligonucleotides in the laboratory and the clinic, The Journal of pathology, 226 (2012) 365-379.

[99] R. Ryther, A. Flynt, J. Phillips, J. Patton, siRNA therapeutics: big potential from small RNAs, Gene therapy, 12 (2005) 5-11.

[100] M. Wilda, U. Fuchs, W. Wössmann, A. Borkhardt, Killing of leukemic cells with a BCR/ABL fusion gene by RNA interference (RNAi), Oncogene, 21 (2002) 5716-5724.

[101] J.-M. Jacque, K. Triques, M. Stevenson, Modulation of HIV-1 replication by RNA interference, Nature, 418 (2002) 435-438.

[102] J. Capodici, K. Karikó, D. Weissman, Inhibition of HIV-1 infection by small interfering RNA-mediated RNA interference, The Journal of Immunology, 169 (2002) 5196-5201.

[103] K. Hamasaki, K. Nakao, K. Matsumoto, T. Ichikawa, H. Ishikawa, K. Eguchi, Short interfering RNA-directed inhibition of hepatitis B virus replication, FEBS letters, 543 (2003) 5154.

[104] J.A. Wilson, S. Jayasena, A. Khvorova, S. Sabatinos, I.G. Rodrigue-Gervais, S. Arya, F. Sarangi, M. Harris-Brandts, S. Beaulieu, C.D. Richardson, RNA interference blocks gene 
expression and RNA synthesis from hepatitis C replicons propagated in human liver cells, Proceedings of the National Academy of Sciences, 100 (2003) 2783-2788.

[105] Q. Ge, M.T. McManus, T. Nguyen, C.-H. Shen, P.A. Sharp, H.N. Eisen, J. Chen, RNA interference of influenza virus production by directly targeting mRNA for degradation and indirectly inhibiting all viral RNA transcription, Proceedings of the National Academy of Sciences, 100 (2003) 2718-2723.

[106] M.J. Wood, B. Trülzsch, A. Abdelgany, D. Beeson, Therapeutic gene silencing in the nervous system, Human molecular genetics, 12 (2003) R279-R284.

[107] D.P. Cioca, Y. Aoki, K. Kiyosawa, RNA interference is a functional pathway with therapeutic potential in human myeloid leukemia cell lines, Cancer Gene Therapy, 10 (2003) 125-133.

[108] F. Czauderna, A. Santel, M. Hinz, M. Fechtner, B. Durieux, G. Fisch, F. Leenders, W. Arnold, K. Giese, A. Klippel, Inducible shRNA expression for application in a prostate cancer mouse model, Nucleic acids research, 31 (2003) e127-e127.

[109] A. Bhoumik, T.-G. Huang, V. Ivanov, L. Gangi, R.F. Qiao, S.L. Woo, S.-H. Chen, Z.e. Ronai, An ATF2-derived peptide sensitizes melanomas to apoptosis and inhibits their growth and metastasis, The Journal of clinical investigation, 110 (2002) 643-650.

[110] G. Yang, J.A. Thompson, B. Fang, J. Liu, Silencing of H-ras gene expression by retrovirus-mediated siRNA decreases transformation efficiency and tumorgrowth in a model of human ovarian cancer, Oncogene, 22 (2003) 5694-5701.

[111] K.A. Whitehead, R. Langer, D.G. Anderson, Knocking down barriers: advances in siRNA delivery, Nature reviews Drug discovery, 8 (2009) 129-138.

[112] A. Tamura, Y. Nagasaki, Smart siRNA delivery systems based on polymeric nanoassemblies and nanoparticles, Nanomedicine, 5 (2010) 1089-1102.

[113] T.G. Park, J.H. Jeong, S.W. Kim, Current status of polymeric gene delivery systems, Advanced drug delivery reviews, 58 (2006) 467-486.

[114] X.-Z. Yang, S. Dou, T.-M. Sun, C.-Q. Mao, H.-X. Wang, J. Wang, Systemic delivery of siRNA with cationic lipid assisted PEG-PLA nanoparticles for cancer therapy, Journal of Controlled Release, 156 (2011) 203-211. 
[115] X. Qian, L. Long, Z. Shi, C. Liu, M. Qiu, J. Sheng, P. Pu, X. Yuan, Y. Ren, C. Kang, Starbranched amphiphilic PLA-b-PDMAEMA copolymers for co-delivery of miR-21 inhibitor and doxorubicin to treat glioma, Biomaterials, 35 (2014) 2322-2335.

[116] N. Cao, D. Cheng, S. Zou, H. Ai, J. Gao, X. Shuai, The synergistic effect of hierarchical assemblies of siRNA and chemotherapeutic drugs co-delivered into hepatic cancer cells, Biomaterials, 32 (2011) 2222-2232.

[117] H.Y. Cho, A. Srinivasan, J. Hong, E. Hsu, S. Liu, A. Shrivats, D. Kwak, A.K. Bohaty, H.j. Paik, J.O. Hollinger, K. Matyjaszewski, Synthesis of Biocompatible PEG-Based Star Polymers with Cationic and Degradable Core for siRNA Delivery, Biomacromolecules, 12 (2011) 34783486.

[118] H. Dong, W. Dai, H. Ju, H. Lu, S. Wang, L. Xu, S.-F. Zhou, Y. Zhang, X. Zhang, Multifunctional Poly(l-lactide)-Polyethylene Glycol-Grafted Graphene Quantum Dots for Intracellular MicroRNA Imaging and Combined Specific-Gene-Targeting Agents Delivery for Improved Therapeutics, ACS Applied Materials \& Interfaces, 7 (2015) 11015-11023.

[119] Y.F. Tan, P. Chandrasekharan, D. Maity, C.X. Yong, K.-H. Chuang, Y. Zhao, S. Wang, J. Ding, S.-S. Feng, Multimodal tumor imaging by iron oxides and quantum dots formulated in poly (lactic acid)-d-alpha-tocopheryl polyethylene glycol 1000 succinate nanoparticles, Biomaterials, 32 (2011) 2969-2978.

[120] V. Bagalkot, L. Zhang, E. Levy-Nissenbaum, S. Jon, P.W. Kantoff, R. Langer, O.C. Farokhzad, Quantum dot-aptamer conjugates for synchronous cancer imaging, therapy, and sensing of drug delivery based on bi-fluorescence resonance energy transfer, Nano letters, 7 (2007) 3065-3070.

[121] F. Ye, Å. Barrefelt, H. Asem, M. Abedi-Valugerdi, I. El-Serafi, M. Saghafian, K. AbuSalah, S. Alrokayan, M. Muhammed, M. Hassan, Biodegradable polymeric vesicles containing magnetic nanoparticles, quantum dots and anticancer drugs for drug delivery and imaging, Biomaterials, 35 (2014) 3885-3894.

[122] W.B. Tan, S. Jiang, Y. Zhang, Quantum-dot based nanoparticles for targeted silencing of HER2/neu gene via RNA interference, Biomaterials, 28 (2007) 1565-1571.

[123] L. Xiao, B. Wang, G. Yang, M. Gauthier, Poly (lactic acid)-based biomaterials: synthesis, modification and applications, INTECH Open Access Publisher, 2012. 
[124] E. Marin, M.I. Briceño, C. Caballero-George, Critical evaluation of biodegradable polymers used in nanodrugs, International journal of nanomedicine, 8 (2013) 3071-3091.

[125] M. Jamshidian, E.A. Tehrany, M. Imran, M. Jacquot, S. Desobry, Poly-Lactic Acid: Production, Applications, Nanocomposites, and Release Studies, Comprehensive Reviews in Food Science and Food Safety, 9 (2010) 552-571.

[126] J.M. Brady, D.E. Cutright, R.A. Miller, G.C. Battistone, E.E. Hunsuck, Resorption rate, route of elimination, and ultrastructure of the implant site of polylactic acid in the abdominal wall of the rat, Journal of Biomedical Materials Research, 7 (1973) 155-166. 